Preprint typeset in JHEP style - HYPER VERSION

SISSA $/ 100 / 03 / \mathrm{EP}$

hep-th/0311198

\title{
Dressed Sliver solutions in Vacuum String Field Theory
}

\author{
L. Bonora, C. Maccaferri, \\ International School for Advanced Studies (SISSA/ISAS) \\ Via Beirut 2-4, 34014 Trieste, Italy, and INFN, Sezione di Trieste \\ E-mail: bonora@sissa.it, maccafer@sissa.it \\ P. Prester \\ Department of Theoretical Physics, Faculty of Science, University of Zagreb \\ Bijenička c. 32, p.p. 331, HR-10002 Zagreb, Croatia \\ E-mail: pprester@phy.hr
}

\begin{abstract}
We consider a new class of solutions (dressed slivers) in Vacuum String Field Theory, which represent D25-branes. For each dressed sliver we introduce a deformation parameter and define a family of states which are characterized by new abelian *subalgebras. We show that this deformation parameter can be used as a regulator: it allows us to define for each such solution a finite norm and energy density. Finally we show how to generalize these results to parallel coincident and to lower dimensional branes.
\end{abstract}




\section{Contents}

1. Introduction 1

2. Dressing the sliver 3

3. A discussion of $\xi \quad 8$

4. The states $\hat{\Xi}_{\epsilon} \quad 10$

5. The dressed sliver action: matter part. 14

6. The ghost dressed sliver 18

6.1 Overall regularized action 22

7. Other finite norm solutions. 22

8. Some comments and conclusions 25

8.1 Conclusions 27

A. A collection of well-known formulae 28

B. Evaluation of determinants 29

B.1 $\operatorname{Det}\left(1-\hat{\mathcal{T}}_{\epsilon} \mathcal{M}\right)$

B.2 $\operatorname{Det}\left(1-\hat{\mathcal{T}}_{\epsilon_{1} \epsilon_{2}} \mathcal{M}\right)$

B.3 $\operatorname{det}\left(1-\hat{T}_{\epsilon}^{2}\right)$

B.4 $\operatorname{det}\left(1-\hat{T}_{\epsilon_{1}} \hat{T}_{\epsilon_{2}}\right)$

G. Limit prescriptions 34

C.1 Double limit 34

C.2 Triple limit 35

D. Derivation of ghost product 36

\section{Introduction}

VSFT, [1], is a version of Witten's open SFT, [2], that is supposed to describe the theory at the minimum of the tachyonic potential. There is evidence that at this point the negative tachyonic potential exactly compensates for the D25-brane tension. No open string mode is expected to be excited, so that the BRST cohomology must be trivial. This state can only correspond to the closed string vacuum. A time-dependent solution which describes 
the evolution from the maximum of the tachyon potential to such a minimum (a rolling tachyon), if it exists, would describe the decay of the D25-brane into closed string states. That such a solution exists has been argued in many ways; in particular this has led to the formulation of a new kind of duality between open and closed strings [3]. Although there have been some attempts to describe such phenomena in a SFT framework [4], no analytical control has been achieved so far.

In this regard VSFT could play an important role. VSFT is a simplified version of SFT. The BRST operator $\mathcal{Q}$ takes a very simple form in terms of ghost oscillators alone. It is clearly simpler to work in such a framework than in the original SFT. In fact many classical solutions have been shown to exist, which are candidates for representing D-branes (the sliver, the butterfly, etc.), and other classical solutions have been found (lump solutions) which may represent lower dimensional D-branes [5, 6, 7, 8, 9, 10, 11]. In some cases the spectrum around such solutions has been analyzed, [13, 14, 15, 16, 17, 18, 19, 20] and there are hints that it provides the modes of a D-brane spectrum. However the responses of VSFT are still far from being satisfactory. There are a series of nontrivial problems left behind. Let us consider for definiteness the sliver solution. To start with it has vanishing action for the matter part and infinite action for the ghost part, but it is impossible to make a finite number out of them, [34. Second, it is not at all clear, at least in the operator formalism, whether the solutions to the linearized equations of motion around the sliver can accommodate all the open string modes (as one would expect if the sliver has to represent a D25-brane). Third, the other Virasoro constraints on such modes are nowhere to be seen.

In the face of this evidence, one can conclude either that VSFT is an oversimplified theory which has lost too many degrees of freedom to describe string physics correctly, or that the sliver is not a very convenient solution to represent a D25-brane. In this paper we take the second point of view: we wish to find a solution to the VSFT equation of motion which is more appropriate than the sliver to represent the D25-brane of open string field theory. On the other hand the sliver has many interesting properties: it is simple and algebraically appealing (it is a squeezed state), its Neumann matrix CS commutes with the twisted matrices of the three strings vertex coefficients and the calculations involving the sliver are relatively simple. Therefore in order to define a new solution we choose to stay as close as possible to the sliver. In practice we start from the sliver and 'perturb it' by adding to $C S$ a suitable rank one projector $P$. We can show not only that this is a solution to the VSFT equations of motion, but that we can define infinite many independent such solutions. We call such solutions dressed slivers.

These new solutions turn out to have an ill-defined $b p z$-norm and action. To remedy this, we multiply the projector $P$ by a real parameter $\epsilon$, thus creating an interpolating family of states between the sliver $(\epsilon=0)$ and the dressed sliver $(\epsilon=1)$. We define a suitable regularization procedure for the determinants that appear in this kind of trade, by means of the level truncation parameter $L$. Then we tune the latter to $1-\epsilon$ and show that the norm and the action corresponding to the dressed sliver can be made finite. A significant part of the paper is devoted to the discussion of the regularization procedure. As it turns out, there are many of them: by imposing the validity of the equation of motion, 
we single out a hopefully consistent regularization scheme.

In a companion paper, 41], the spectrum of states around the dressed sliver is analyzed. It will be seen there that this new type of solution solves several problems the spectrum around the sliver has.

The paper is organized as follows. In section 2, after a brief summary concerning the operatorial construction of the sliver in the matter sector, we define the dressed sliver state by using a "half string vector" $\xi$; we show that such a state solves the zero momentum projector equations. Section 3 is devoted to the concrete construction of such a vector $\xi$ : we show that there are infinite many numerable consistent choices that solve the projector equation. In section 4 we implement a deformation procedure, which exhibits many interesting features: first of all such deformation turns out to define a continuous family of states which is closed under the $*$-product; we show that, in such a family, the $*$-product acts in a commutative way and that the dressed sliver is the identity element, inverse elements and null elements are shown to exist: we show that we can consistently extract two abelian subalgebras which are isomorphic via an inversion map, each one containing an identity element and a null element as projectors. Section 5 deals with the computation of the action of the dressed sliver, we implement a regularization relating the vanishing of the determinants involving infinite level Neumann coefficients to infinities which arise due to dressing. We show that it is possible to extract a finite answer for the action by appropriately choosing a limiting procedure. In section 6 we extend our results to the ghost part in a straightforward way, and show that also the ghost part of the action can be made finite. In section 8 some other finite-action solutions are discussed, in particular the ones related to parallel coincident and lower dimensional D-branes. In section 9 some relations with previous works are discussed and some concluding remarks are presented. Appendix A contains well known properties of matter and ghost star algebra. Appendix B contains detailed calculations of the determinants needed in the main text. Appendix $\mathrm{C}$ deals with subtleties of the limiting procedures which arise while regularizing the solutions. Appendix D contains an explicit proof concerning the $*$-product of dressed states in the ghost sector.

\section{Dressing the sliver}

To start with we recall some formulas relevant to VSFT. The action is

$$
\mathcal{S}(\Psi)=-\frac{1}{g_{0}^{2}}\left(\frac{1}{2}\langle\Psi|\mathcal{Q}| \Psi\rangle+\frac{1}{3}\langle\Psi \mid \Psi * \Psi\rangle\right)
$$

where

$$
\mathcal{Q}=c_{0}+\sum_{n>0}(-1)^{n}\left(c_{2 n}+c_{-2 n}\right)
$$

Notice that the action (2.1) does not contain any singular normalization constant, as opposed to 10, 13]. This important issue will be discussed in section 8 . The equation of motion is

$$
\mathcal{Q} \Psi=-\Psi * \Psi
$$


and the ansatz for nonperturbative solutions is in the factorized form

$$
\Psi=\Psi_{m} \otimes \Psi_{g}
$$

where $\Psi_{g}$ and $\Psi_{m}$ depend purely on ghost and matter degrees of freedom, respectively. Then eq.(2.3) splits into

$$
\begin{aligned}
\mathcal{Q} \Psi_{g} & =-\Psi_{g} *_{g} \Psi_{g} \\
\Psi_{m} & =\Psi_{m} *_{m} \Psi_{m}
\end{aligned}
$$

where $*_{g}$ and $*_{m}$ refers to the star product involving only the ghost and matter part.

The action for this type of solution becomes

$$
\mathcal{S}(\Psi)=-\frac{1}{6 g_{0}^{2}}\left\langle\Psi_{g}|\mathcal{Q}| \Psi_{g}\right\rangle\left\langle\Psi_{m} \mid \Psi_{m}\right\rangle
$$

$\left\langle\Psi_{m} \mid \Psi_{m}\right\rangle$ is the ordinary inner product, $\left\langle\Psi_{m}\right|$ being the $b p z$ conjugate of $\left|\Psi_{m}\right\rangle$ (see below).

We shall see later on how to find solutions to (2.5). For the time being, as an introduction to the problem, let us concentrate on the matter part, eq.(2.6). The $*_{m}$ product is defined as follows

$$
{ }_{123}\left\langle V_{3} \mid \Psi_{1}\right\rangle_{1}\left|\Psi_{2}\right\rangle_{2}={ }_{3}\left\langle\Psi_{1} *_{m} \Psi_{2}\right|
$$

where the three strings vertex $V_{3}$ is

$$
\left|V_{3}\right\rangle_{123}=\int d^{26} p_{(1)} d^{26} p_{(2)} d^{26} p_{(3)} \delta^{26}\left(p_{(1)}+p_{(2)}+p_{(3)}\right) \exp (-E)|0, p\rangle_{123}
$$

with

$$
E=\sum_{a, b=1}^{3}\left(\frac{1}{2} \sum_{m, n \geq 1} \eta_{\mu \nu} a_{m}^{(a) \mu \dagger} V_{m n}^{a b} a_{n}^{(b) \nu \dagger}+\sum_{n \geq 1} \eta_{\mu \nu} p_{(a)}^{\mu} V_{0 n}^{a b} a_{n}^{(b) \nu \dagger}+\frac{1}{2} \eta_{\mu \nu} p_{(a)}^{\mu} V_{00}^{a b} p_{(b)}^{\nu}\right)
$$

Summation over the Lorentz indices $\mu, \nu=0, \ldots, 25$ is understood and $\eta$ denotes the flat Lorentz metric. The operators $a_{m}^{(a) \mu}, a_{m}^{(a) \mu \dagger}$ denote the non-zero modes matter oscillators of the $a$-th string, which satisfy

$$
\left[a_{m}^{(a) \mu}, a_{n}^{(b) \nu \dagger}\right]=\eta^{\mu \nu} \delta_{m n} \delta^{a b}, \quad m, n \geq 1
$$

$p_{(r)}$ is the momentum of the $a$-th string and $|0, p\rangle_{123} \equiv\left|p_{(1)}\right\rangle \otimes\left|p_{(2)}\right\rangle \otimes\left|p_{(3)}\right\rangle$ is the tensor product of the Fock vacuum states relative to the three strings with definite c.m. momentum . $\left|p_{(a)}\right\rangle$ is annihilated by the annihilation operators $a_{m}^{(a) \mu}(m \geq 1)$ and it is eigenstate of the momentum operator $\hat{p}_{(a)}^{\mu}$ with eigenvalue $p_{(a)}^{\mu}$. The normalization is

$$
\left\langle p_{(a)} \mid p_{(b)}^{\prime}\right\rangle=\delta_{a b} \delta^{26}\left(p+p^{\prime}\right)
$$

The symbols $V_{n m}^{a b}, V_{0 m}^{a b}, V_{00}^{a b}$ will denote the coefficients computed in [24, 25, 26, 27, 28, 29]. We will use them in the notation of Appendix A and B of [7]. 
To complete the definition of the $*_{m}$ product we must specify the $b p z$ conjugation properties of the oscillators

$$
b p z\left(a_{n}^{(a) \mu}\right)=(-1)^{n+1} a_{-n}^{(a) \mu}
$$

In this paper we will mostly discuss solutions representing D25-branes (see a comment on lower dimensional branes at the end), which are translationally invariant. As a consequence we set all the momenta to zero. So the integration over the momenta will be dropped and the only surviving part in $E$ will be the one involving $V_{n m}^{a b}$. This is what we understand in the following by $*_{m}$, unless otherwise specified.

Let us now return to eq.(2.6). Its solutions are projectors of the $*_{m}$ algebra. We recall the simplest one, the sliver. It is defined by

$$
|\Xi\rangle=\mathcal{N} e^{-\frac{1}{2} a^{\dagger} S a^{\dagger}}|0\rangle, \quad a^{\dagger} S a^{\dagger}=\sum_{n, m=1}^{\infty} a_{n}^{\mu \dagger} S_{n m} a_{m}^{\nu \dagger} \eta_{\mu \nu}
$$

This state satisfies eq.(2.6) provided the matrix $S$ satisfies the equation

$$
S=V^{11}+\left(V^{12}, V^{21}\right)(1-\Sigma \mathcal{V})^{-1} \Sigma\left(\begin{array}{l}
V^{21} \\
V^{12}
\end{array}\right)
$$

where

$$
\Sigma=\left(\begin{array}{cc}
S & 0 \\
0 & S
\end{array}\right), \quad \mathcal{V}=\left(\begin{array}{ll}
V^{11} & V^{12} \\
V^{21} & V^{22}
\end{array}\right)
$$

The proof of this fact is well-known. First one expresses eq.(2.16) in terms of the twisted matrices $X=C V^{11}, X_{+}=C V^{12}$ and $X_{-}=C V^{21}$, together with $T=C S=S C$, where $C_{n m}=(-1)^{n} \delta_{n m}$. The matrices $X, X_{+}, X_{-}$are mutually commuting. Then, requiring that $T$ commute with them as well, one can show that eq.2.16) reduces to the algebraic equation

$$
X T^{2}-(1+X) T+X=0
$$

The interesting solution is

$$
T=\frac{1}{2 X}(1+X-\sqrt{(1+3 X)(1-X)})
$$

The normalization constant $\mathcal{N}$ is calculated to be

$$
\mathcal{N}=(\operatorname{Det}(1-\Sigma \mathcal{V}))^{\frac{D}{2}}
$$

where $D=26$. The contribution of the sliver to the matter part of the action (see (2.7)) is given by

$$
\langle\Xi \mid \Xi\rangle=\frac{\mathcal{N}^{2}}{\left(\operatorname{det}\left(1-S^{2}\right)\right)^{\frac{D}{2}}}
$$

Both eq.(2.19) and (2.20) are ill-defined and need to be regularized, after which they both turn out to vanish. This subject will be taken up again in section 5 .

In Appendix A we collect a series of properties and results concerning the matrices $X, X_{-}, X_{+}, T$, together with other formulas that will be needed in the following. 
Now we want to deform the sliver by adding some special matrix to $S$. To this end we first introduce the infinite vector $\xi=\left\{\xi_{n}\right\}$ which is chosen to satisfy the condition

$$
\rho_{1} \xi=0, \quad \rho_{2} \xi=\xi
$$

Notice that this vector does not have any Lorentz label (compare with [8]). Next we set

$$
\xi^{T} \frac{1}{1-T^{2}} \xi=1, \quad \xi^{T} \frac{T}{1-T^{2}} \xi=\kappa
$$

where $^{T}$ denotes matrix transposition. Eqs.(2.22) will be studied in section 3. Our candidate for the dressed sliver solution is given by an ansatz similar to (2.14)

$$
|\hat{\Xi}\rangle=\hat{\mathcal{N}} e^{-\frac{1}{2} a^{\dagger} \hat{S} a^{\dagger}}|0\rangle,
$$

with $S$ replaced by

$$
\hat{S}=S+R, \quad R_{n m}=\frac{1}{\kappa+1}\left(\xi_{n}(-1)^{m} \xi_{m}+\xi_{m}(-1)^{n} \xi_{n}\right)
$$

As a consequence $T$ is replaced by

$$
\hat{T}=T+P, \quad P_{n m}=\frac{1}{\kappa+1}\left(\xi_{m} \xi_{n}+\xi_{n}(-1)^{m+n} \xi_{m}\right)
$$

From time to time a bra and ket notation will be used to represent $P$ :

$$
P=\frac{1}{\kappa+1}(|\xi\rangle\langle\xi|+| C \xi\rangle\langle C \xi|)
$$

We require the dressed sliver to satisfy hermiticity, which amounts to imposing that the $b p z-$ conjugate state coincide with the hermitean conjugate one. This in turn implies

$$
|\xi\rangle\langle C \xi|+| \xi\rangle\left\langle C \xi|=| \xi^{*}\right\rangle\left\langle C \xi^{*}|+| \xi^{*}\right\rangle\left\langle C \xi^{*}\right|
$$

We satisfy this condition by choosing $\xi$ real. This means that $\kappa$ is real (and negative). We remark at this point that the conditions (2.22) are not very stringent. The only thing one has to worry is that the lhs's are finite (this is the only true condition). Once this is true the rest follows from suitably rescaling $\xi$, so that the first equation is satisfied, and from the reality of $\xi$ (see also next section).

We claim that $|\hat{\Xi}\rangle$ is a projector. The dressed sliver matrix $\hat{T}$ does not commute with $X, X_{-}, X_{+}$(as T does), but we can nevertheless make use of the property $C \hat{T}=\hat{T} C$, because $C P=P C$. To prove our claim we must show that

$$
V^{11}+\left(V^{12}, V^{21}\right)(1-\hat{\Sigma} \mathcal{V})^{-1} \Sigma\left(\begin{array}{l}
V^{21} \\
V^{12}
\end{array}\right)=\hat{S}
$$

where

$$
\hat{\Sigma}=\left(\begin{array}{cc}
\hat{S} & 0 \\
0 & \hat{S}
\end{array}\right)
$$


We will in fact prove in detail that

$$
X+\left(X_{+}, X_{-}\right)(1-\hat{\mathcal{T}} \mathcal{M})^{-1} \hat{\mathcal{T}}\left(\begin{array}{l}
X_{-} \\
X_{+}
\end{array}\right)=\hat{T}
$$

where

$$
\hat{\mathcal{T}}=C \hat{\Sigma}=\mathcal{T}+\mathcal{P}, \quad \mathcal{M}=C \mathcal{V}
$$

To this end, let us define

$$
\hat{\mathcal{K}}=1-\hat{\mathcal{T}} \mathcal{M}=1-\mathcal{T} \mathcal{M}-\mathcal{P} \mathcal{M}=\mathcal{K}-\mathcal{P} \mathcal{M}
$$

The symbol $\mathcal{K}$ is the same as $\hat{\mathcal{K}}$ when the deformation $P$ is absent, so it is the quantity relevant to the sliver. Now we write

$$
\hat{\mathcal{K}}^{-1}=(1-\hat{\mathcal{T}} \mathcal{M})^{-1}=\mathcal{K}^{-1}\left(1-\mathcal{P} \mathcal{M} \mathcal{K}^{-1}\right)^{-1}
$$

We have

$$
\left(1-\mathcal{P} \mathcal{M K}^{-1}\right)^{-1} \mathcal{P}=\left(\begin{array}{cc}
1 & \rho_{1}-\kappa \rho_{2} \\
\rho_{2}-\kappa \rho_{1} & 1
\end{array}\right) \mathcal{P}
$$

This can be shown either by expanding the lhs in power series or multiplying this equation from the left by $1-\mathcal{P} \mathcal{M} \mathcal{K}^{-1}$ and verifying that it is an identity. To obtain this result one must use eq.(2.22) and the formulas in Appendix A, from which in particular one can derive

$$
X_{+} \xi=X(T-1) \xi, \quad X_{-} \xi=(1-X T) \xi
$$

Now we can evaluate the lhs of eq.2.29

$$
\begin{aligned}
& X+\left(X_{+}, X_{-}\right)(1-\hat{\mathcal{T}} \mathcal{M})^{-1} \hat{\mathcal{T}}\left(\begin{array}{c}
X_{-} \\
X_{+}
\end{array}\right) \\
& =X+\left(X_{+}, X_{-}\right) \mathcal{K}^{-1}\left(1-\mathcal{P} \mathcal{M} \mathcal{K}^{-1}\right)^{-1}(\mathcal{T}+\mathcal{P})\left(\begin{array}{c}
X_{-} \\
X_{+}
\end{array}\right) \\
& =X+\left(\rho_{1}, \rho_{2}\right) \mathcal{T}\left(\begin{array}{c}
X_{-} \\
X_{+}
\end{array}\right)+\left(\rho_{1}, \rho_{2}\right)\left(1-\mathcal{P} \mathcal{M} \mathcal{K}^{-1}\right)^{-1} \mathcal{P}\left(\begin{array}{c}
X_{-} \\
X_{+}
\end{array}\right) \\
& \quad+\left(\rho_{1}, \rho_{2}\right)\left(1-\mathcal{P} \mathcal{M} \mathcal{K}^{-1}\right)^{-1} \mathcal{P} \mathcal{M} \mathcal{K}^{-1} \mathcal{T}\left(\begin{array}{c}
X_{-} \\
X_{+}
\end{array}\right)
\end{aligned}
$$

The first two terms in the $r h s$ are exactly $T$. Next one notices that

$$
\left(\rho_{1}, \rho_{2}\right)\left(1-\mathcal{P} \mathcal{M} \mathcal{K}^{-1}\right)^{-1}=(1,1)
$$

Therefore

$$
\begin{aligned}
& X+\left(X_{+}, X_{-}\right) \hat{\mathcal{K}}^{-1} \hat{\mathcal{T}}\left(\begin{array}{c}
X_{-} \\
X_{+}
\end{array}\right)=T+P X_{-}+P X_{+}+(1,1) P\left(\begin{array}{c}
T X \rho_{2}+T X_{+} \rho_{1} \\
T X_{-} \rho_{2}+T X \rho_{1}
\end{array}\right)= \\
& =T+\frac{1}{\kappa+1}(|C \xi\rangle\langle C \xi|X(T-1)+| \xi\rangle\langle\xi|(1-X T)+| C \xi\rangle\langle C \xi|(1-X T)+| \xi\rangle\langle\xi| X(T-1)) \\
& \quad+\frac{1}{\kappa+1}(|C \xi\rangle\langle C \xi|T(1-X T)+| \xi\rangle\langle\xi|X T+| C \xi\rangle\langle C \xi|X T+| \xi\rangle\langle\xi| T(1-X T))= \\
& =T+\frac{1}{\kappa+1}(|\xi\rangle\langle\xi|+| C \xi\rangle\langle C \xi|)=T+P=\hat{T}
\end{aligned}
$$


In the passage to the last line we have used the identity $X T-X+T-X T^{2}=0$. This completes the proof that $\hat{\Xi}$ is a solution to 2.6 .

We remark that, due to the arbitrariness of $\xi$, the result we have obtained brings into the game an infinite family of solutions to the equations of motion ${ }^{1}$. We shall see later that this result can be easily generalized. For the time being however we are interested in studying the properties of these new solutions.

The normalization constant $\hat{\mathcal{N}}$ is given by (see appendix B)

$$
\hat{\mathcal{N}}=\operatorname{Det}(1-\hat{\Sigma} \mathcal{V})^{\frac{D}{2}}=\operatorname{Det}(1-\mathcal{T} \mathcal{M})^{\frac{D}{2}} \operatorname{Det}\left(1-\mathcal{P} \mathcal{M} K^{-1}\right)^{\frac{D}{2}}=\operatorname{Det}(1-\mathcal{T} \mathcal{M})^{\frac{D}{2}} \cdot \frac{1}{(\kappa+1)^{D}}
$$

However, if one tries to compute the norm of this state (which corresponds to the its contribution to the action), i.e. $\langle\hat{\Xi} \mid \hat{\Xi}\rangle$, one finds an indeterminate result (as will be apparent from the calculation in section 5). It is evident that we have to introduce a regulator in order to end up with a finite action. Our idea is to introduce a numerical parameter $\epsilon$ in front of $R$ in the definition of $\hat{\Xi}$. In this way we define new squeezed states $\hat{\Xi}_{\epsilon}$ characterized by the matrix $\hat{S}_{\epsilon}=S+\epsilon R$. But, before we come to that, a discussion of some issues concerning the vector $\xi$ is in order.

\section{A discussion of $\xi$}

In this section we will give a precise construction of the "half string" vector $\xi$. In so doing it is very convenient to use the continuous $k$ basis of the star algebra.

In 222 it was shown that the Neumann coefficients $\left(X, X_{+}, X_{-}\right)$can be simultaneusly put in a continuous diagonal form as follows

$$
X=\int_{-\infty}^{\infty} d k x(k)|k\rangle\left\langle k\left|, \quad X_{ \pm}=\int_{-\infty}^{\infty} d k x_{ \pm}(k)\right| k\right\rangle\langle k|
$$

The eigenvalues are given $b y^{2}$

$$
\begin{aligned}
x(k) & =-\frac{1}{1+2 \cosh \left(\frac{\pi k}{2}\right)} \\
x_{ \pm}(k) & =\frac{1+\cosh \left(\frac{\pi k}{2}\right) \pm \sinh \left(\frac{\pi k}{2}\right)}{1+2 \cosh \left(\frac{\pi k}{2}\right)}
\end{aligned}
$$

and the eigenvectors

$$
\begin{aligned}
|k\rangle & =\left(\frac{2}{k} \sinh \left(\frac{\pi k}{2}\right)\right)^{-\frac{1}{2}} \sum_{n=1}^{\infty} v_{n}(k)|n\rangle \\
v_{n}(k) & =\sqrt{n} \oint \frac{d z}{2 \pi i} \frac{1}{z^{n+1}} \frac{1}{k}\left(1-e^{-k \tan ^{-1}(z)}\right)
\end{aligned}
$$

These eigenvectors are normalized by the condition, [34],

$$
\left\langle k \mid k^{\prime}\right\rangle=\delta\left(k-k^{\prime}\right)
$$

\footnotetext{
${ }^{1}$ We believe this multiplicity of solutions to correspond mostly to gauge degrees of freedom.

${ }^{2}$ We hope the reader should not confuse $k$ with $\kappa$.
} 
In this basis the sliver matrix $T$ takes the remarkably simple form

$$
T=-\int_{-\infty}^{\infty} d k e^{-\frac{\pi|k|}{2}}|k\rangle\langle k|
$$

One should think at the real line spanned by $k$ as a parametrization of the string itself in which the midpoint is represented by the $k=0$ eigenvector and the left (right) half by $k>0(k<0)$. This is easy to see once the form of the projectors $\rho_{1}, \rho_{2}$ is given in such a basis

$$
\begin{aligned}
& \rho_{1}=\int_{-\infty}^{\infty} d k \theta(k)|k\rangle\left\langle k\left|=\int_{0}^{\infty} d k\right| k\right\rangle\langle k| \\
& \rho_{2}=\int_{-\infty}^{\infty} d k \theta(-k)|k\rangle\left\langle k\left|=\int_{0}^{\infty} d k\right|-k\right\rangle\langle-k|
\end{aligned}
$$

The value of these projectors at $k=0$ is a subtle point [18] and we will avoid this singular mode in the constrution of the vector $\xi$. Since $\xi$ is constrained by $\rho_{2}|\xi\rangle=|\xi\rangle, \rho_{1}|\xi\rangle=0$, it is natural to parametrize it as

$$
|\xi\rangle=\int_{0}^{\infty} d k \xi(y)|-k\rangle
$$

where $y=\frac{\pi k}{2}$. Now the vector $|\xi\rangle$ is represented by the function $\xi(y)$, which has support on the positive real axis. The expressions (2.22) take the integral form

$$
\begin{aligned}
& \left\langle\xi\left|\frac{1}{1-T^{2}}\right| \xi\right\rangle=\frac{2}{\pi} \int_{0}^{\infty} d y \xi^{2}(y) \frac{1}{1-e^{-2 y}}=1 \\
& \left\langle\xi\left|\frac{T}{1-T^{2}}\right| \xi\right\rangle=\frac{2}{\pi} \int_{0}^{\infty} d y \xi^{2}(y) \frac{-e^{-y}}{1-e^{-2 y}}=\kappa
\end{aligned}
$$

Note that the denominator $1-T^{2}$ vanishes at $k=0$, so, in order to avoid infinities, we further require $\xi(y)$ to vanish rapidly enough at $y=0$. This means that the vector $\xi$ does not excite the (zero momentum) midpoint mode. The space of functions with support on the positive axis, vanishing at the origin, and satisfying (3.4, 3.5) with finite $\kappa$, are spanned by a (numerable) infinite set of "orthogonal" functions defined by

$$
\xi_{n}(y)=\left(\frac{\pi}{2}\left(1-e^{-2 y}\right) e^{-y}\right)^{\frac{1}{2}} L_{n}(y)
$$

where $L_{n}(y)$ is the $n$-th Laguerre polynomial.

The normalization factor in front of the polynomials has been chosen in order to satisfy

$$
\left\langle\xi_{n}\left|\frac{1}{1-T^{2}}\right| \xi_{m}\right\rangle=\int_{0}^{\infty} d y e^{-y} L_{n}(y) L_{m}(y)=\delta_{n m}
$$

In a similar fashion, using standard properties of Laguerre polynomials ${ }^{3}$, one can prove that

$$
\left\langle\xi_{n}\left|\frac{T}{1-T^{2}}\right| \xi_{m}\right\rangle=-\int_{0}^{\infty} d y e^{-2 y} L_{n}(y) L_{m}(y)=K_{n m}=-\frac{1}{2^{n+m}} \frac{(m+n) !}{n ! m !}
$$

\footnotetext{
${ }^{3}$ In particular we need the relation$$
L_{n}(\lambda y)=\sum_{p=0}^{n}\left(\begin{array}{l}
n \\
p
\end{array}\right) \lambda^{n-p}(1-\lambda)^{p} L_{n-p}(y)
$$ 
A simple numerical analysis shows that the eigenvalues of the matrix $K_{n m}$ lie in the interval $(-1,0)$. This is of course what one should expect once the normalization condition $\left\langle\xi \frac{1}{1-T^{2}} \xi\right\rangle=1$ is imposed. In fact the condition (3.5) differs from (3.4) by the insertion of the matrix $T$, which has a spectrum covering (twice) the interval $(-1,0)$.

In order to prove that these half string vectors can be concretely defined as Fock space vectors, we shall see that it is possible to have a complete control on their norm as well, and that such norms are always positive. Using the same standard manipulations as before, we have

$$
\begin{aligned}
& \left\langle\xi_{n} \mid \xi_{m}\right\rangle=\left\langle\xi_{n}\left|\frac{1-T^{2}}{1-T^{2}}\right| \xi_{m}\right\rangle= \\
& =\int_{0}^{\infty} d y e^{-y}\left(1-e^{-2 y}\right) L_{n}(y) L_{m}(y)=\delta_{n m}-\frac{2^{m-n}}{3^{n+m+1}} \sum_{p=0}^{n} 4^{p}\left(\begin{array}{c}
n \\
p
\end{array}\right)\left(\begin{array}{c}
m \\
m-n+p
\end{array}\right)
\end{aligned}
$$

Again a simple numerical analysis shows that the eigenvalues of the matrix defined by the rhs of (3.9), lie in the interval $(0,1)$ : this definitely ensures the existence of such vectors. As we will see in the last section, we can build orthogonal projectors (in the sense of the star product and of the bpz-norm) by simply using different and orthogonal half-string vectors, where orthogonality is understood in the following sense

$$
\left\langle\xi\left|\frac{1}{1-T^{2}}\right| \xi^{\prime}\right\rangle=0, \quad\left\langle\xi\left|\frac{T}{1-T^{2}}\right| \xi^{\prime}\right\rangle=0,
$$

In view of the above discussion it is obvious that one can always find a finite number of $\xi_{n}$ 's to construct any given number of mutually orthogonal vectors although the number of $\xi_{n}$ 's needed increases faster with respect to the number of orthogonal projectors.

\section{The states $\hat{\Xi}_{\epsilon}$}

After the digression of the previous section, let us return to the problem of regularizing the norm for the matter part of the dressed sliver. As anticipated in section 2, the (naive) definition (2.23) given in section 2 for the dressed sliver does not avoid ambiguities and indefiniteness, when we come to compute its norm. The determinants involved in such calculations are in general not well-defined. To evade this problem our idea is to deform the dressed sliver by introducing a parameter $\epsilon$, so that we get the dressed sliver when $\epsilon=1$. When $\epsilon \neq 1$ the state we obtain is in general not a $*$-algebra projector. We will define the norm of the dressed sliver as the limit of a sequence of such states.

Let us introduce the state

$$
\left|\hat{\Xi}_{\epsilon}\right\rangle=\hat{\mathcal{N}}_{\epsilon} e^{-\frac{1}{2} a^{\dagger} \hat{S}_{\epsilon} a^{\dagger}}|0\rangle,
$$

where

$$
\hat{S}_{\epsilon}=S+\epsilon R,
$$

As a consequence $T$ is replaced by

$$
\hat{T}_{\epsilon}=T+\epsilon P,
$$


The states defined in this way are not in general projectors, but have very interesting properties. Not all of them are needed for the following developments in this paper. However it is worth to make a short detour to illustrate them.

We would like to show that the states (4.1) define a continuous $*$-abelian 1-parameter family of states. First we show that they are closed under the $*-$ product. Hence let us consider

$$
\left|\hat{\Xi}_{\epsilon_{1}}\right\rangle *\left|\hat{\Xi}_{\epsilon_{2}}\right\rangle=\hat{\mathcal{N}}\left(\epsilon_{1}, \epsilon_{2}\right) e^{-\frac{1}{2} a^{\dagger} C\left(T+\epsilon_{1} P\right) *\left(T+\epsilon_{2} P\right) a^{\dagger}}|0\rangle
$$

where we denote

$$
\left(T+\epsilon_{1} P\right) *\left(T+\epsilon_{2} P\right) \equiv X+\left(X_{+}, X_{-}\right)\left(1-\hat{\mathcal{T}}_{\epsilon_{1} \epsilon_{2}} \mathcal{M}\right)^{-1} \hat{\mathcal{T}}_{\epsilon_{1} \epsilon_{2}}\left(\begin{array}{c}
X_{-} \\
X_{+}
\end{array}\right)
$$

and

$$
\hat{\mathcal{T}}_{\epsilon_{1} \epsilon_{2}} \equiv\left(\begin{array}{cc}
\hat{T}_{\epsilon_{1}} & 0 \\
0 & \hat{T}_{\epsilon_{2}}
\end{array}\right)
$$

In order to compute this expression we need the generalized formula

$$
\left(1-\mathcal{P}_{\epsilon_{1} \epsilon_{2}} \mathcal{M K}^{-1}\right)^{-1} \mathcal{P}_{\epsilon_{1} \epsilon_{2}}=\frac{1}{1+\left(1-\epsilon_{1}\right)\left(1-\epsilon_{2}\right) \kappa}\left(\begin{array}{cc}
\left(1-\epsilon_{2}\right) \kappa+1 & \epsilon_{1}\left(\rho_{1}-\kappa \rho_{2}\right) \\
\epsilon_{2}\left(\rho_{2}-\kappa \rho_{1}\right) & \left(1-\epsilon_{1}\right) \kappa+1
\end{array}\right) \mathcal{P}_{\epsilon_{1} \epsilon_{2}}
$$

One can prove this formula as an easy generalization of section 2. Alternatively one can check it directly by multiplying it on the left with $\left(1-\mathcal{P}_{\epsilon_{1} \epsilon_{2}} \mathcal{M} \mathcal{K}^{-1}\right)$ (a detailed proof can be found in the appendix B). Then things are straightforward and we get

$$
\left(T+\epsilon_{1} P\right) *\left(T+\epsilon_{2} P\right)=T+\left(\epsilon_{1} \star \epsilon_{2}\right) P
$$

where we have defined the abelian multiplication law between real numbers

$$
\epsilon_{1} \star \epsilon_{2}=\frac{\epsilon_{1} \epsilon_{2}}{1+\left(1-\epsilon_{1}\right)\left(1-\epsilon_{2}\right) \kappa}
$$

This product is easily shown to be associative

$$
\begin{aligned}
\left(\epsilon_{1} \star \epsilon_{2}\right) \star \epsilon_{3} & =\epsilon_{1} \star\left(\epsilon_{2} \star \epsilon_{3}\right)= \\
& =\frac{\epsilon_{1} \epsilon_{2} \epsilon_{3}}{1+\kappa\left(2-\epsilon_{1}-\epsilon_{2}-\epsilon_{3}+\epsilon_{1} \epsilon_{2} \epsilon_{3}+\kappa\left(1-\epsilon_{1}\right)\left(1-\epsilon_{2}\right)\left(1-\epsilon_{3}\right)\right)}
\end{aligned}
$$

and exhibits three idempotent elements

$$
0 \star 0=0, \quad 1 \star 1=1, \quad \frac{\kappa+1}{\kappa} \star \frac{\kappa+1}{\kappa}=\frac{\kappa+1}{\kappa}
$$

Note that 1 is the identity

$$
\epsilon \star 1=1 \star \epsilon=\epsilon
$$

The inverse with respect to this product is given by

$$
\epsilon^{\star-1}=\frac{(1-\epsilon) \kappa+1}{(1-\epsilon) \kappa+\epsilon}
$$


so that

$$
\epsilon \star \frac{(1-\epsilon) \kappa+1}{(1-\epsilon) \kappa+\epsilon}=1
$$

We have two distinct null elements which are 0 and $\frac{\kappa+1}{\kappa}$

$$
0 \star \epsilon=\epsilon \star 0=0, \quad \frac{\kappa+1}{\kappa} \star \epsilon=\epsilon \star \frac{\kappa+1}{\kappa}=\frac{\kappa+1}{\kappa}
$$

The point $\{\infty\}$ is naturally in the domain as it can be reached from any $\epsilon \neq 0,1, \frac{\kappa+1}{\kappa}$ by $\star$-product

$$
\epsilon \star\left(1+\frac{1}{(1-\epsilon) \kappa}\right)=\infty
$$

The simultaneus presence of two null elements makes their product ambiguous

$$
\frac{\kappa+1}{\kappa} \star 0=\text { indeterminate }
$$

Note in particular that we have

$$
0^{\star-1}=\frac{\kappa+1}{\kappa}, \quad\left(\frac{\kappa+1}{\kappa}\right)^{\star-1}=0
$$

This is very reminescent of what happens with real numbers when they are completed with $\infty$, in which case what is ambiguous is the product $0 \cdot \infty$. One should note actually that this is the same situation, deformed by the parameter $\kappa$, as in the limit $\kappa \rightarrow 0$ one recovers the usual product and, in particular, $\frac{\kappa+1}{\kappa} \rightarrow \infty$.

In view of the structure we have found, two new abelian subalgebra of the $*$-product are naturally identified. The first is $R \cup\{\infty\} \backslash\left\{\frac{\kappa+1}{\kappa}\right\}$ and contains, as projectors, the sliver $(\epsilon=0)$ and the dressed sliver $(\epsilon=1)$. The second is $R \cup\{\infty\} \backslash\{0\}$ and contains the projectors $\epsilon=1$ and $\epsilon=\frac{\kappa+1}{\kappa}$. We will call the state identified by $\epsilon=\frac{\kappa+1}{\kappa}$ the exotic dressed sliver. Note also that these two algebras are isomorphic to each other via the inversion $\operatorname{map}(4.11)$.

Since we are dealing with projectors, normalization is needed. The normalization of all the states in the two algebras is completely fixed once we ask the sliver and the exotic sliver to be really null elements. A general element of the two algebras can be written as

$$
\left|\hat{\Xi}_{\epsilon}\right\rangle^{(1,2)}=\mathcal{N}_{\epsilon}^{(1,2)} e^{-\frac{\epsilon x}{\kappa+1}}|\Xi\rangle
$$

where $|\Xi\rangle$ is the usual sliver with its (vanishing) normalization and the superscript ${ }^{(1,2)}$ labels the algebras, moreover we have identified

$$
x=a_{\mu}^{\dagger} C(|\xi\rangle\langle\xi|+| C \xi\rangle\langle C \xi|) a_{\nu}^{\dagger} \eta^{\mu \nu} .
$$

It is then easy to show that the star products of two such states is given by

$$
\left|\hat{\Xi}_{\epsilon}\right\rangle^{(1,2)} *\left|\hat{\Xi}_{\eta}\right\rangle^{(1,2)}=\frac{\mathcal{N}_{\epsilon}^{(1,2)} \mathcal{N}_{\eta}^{(1,2)}}{\mathcal{N}_{\epsilon \star \eta}^{(1,2)}}\left(\frac{\kappa+1}{1+(1-\epsilon)(1-\eta) \kappa}\right)^{D}\left|\hat{\Xi}_{\epsilon \star \eta}\right\rangle^{(1,2)}
$$


The second factor in the rhs comes from $\operatorname{Det}\left(1-\hat{\mathcal{T}}_{\epsilon \eta} \mathcal{M}\right)^{-\frac{1}{2}}$, see appendix B. In the first algebra the null element is the sliver $(\epsilon=0)$ and, of course, $\mathcal{N}_{0}^{(1)}=1$ since the sliver is a projector by itself. The star product with another state of the same algebra is then

$$
\left|\hat{\Xi}_{0}\right\rangle^{(1)} *\left|\hat{\Xi}_{\epsilon}\right\rangle^{(1)}=\mathcal{N}_{\epsilon}^{(1)}\left(\frac{\kappa+1}{1+(1-\epsilon) \kappa}\right)^{D}\left|\hat{\Xi}_{0}\right\rangle^{(1)}
$$

which implies

$$
\mathcal{N}_{\epsilon}^{(1)}=\left(\frac{1+(1-\epsilon) \kappa}{\kappa+1}\right)^{D}
$$

With this choice of normalization we have, use eq.(4.9),

$$
\frac{\mathcal{N}_{\epsilon}^{(1)} \mathcal{N}_{\eta}^{(1)}}{\mathcal{N}_{\epsilon \star \eta}^{(1)}}=\left(\frac{1+(1-\epsilon)(1-\eta) \kappa}{\kappa+1}\right)^{D}
$$

so the first algebra closes with structure constant 1 ,

$$
\left|\hat{\Xi}_{\epsilon}\right\rangle^{(1)} *\left|\hat{\Xi}_{\eta}\right\rangle^{(1)}=\left|\hat{\Xi}_{\epsilon \star \eta}\right\rangle^{(1)}
$$

Note that the exotic sliver has, in this algebra, an extra vanishing normalization due to the dressing factor, (4.14), so it is naturally excluded. Concerning the inverse algebra one has first to note that, in order for the exotic sliver to be a projector it should be that

$$
\mathcal{N}_{\frac{\kappa+1}{\kappa}}^{(2)}=\frac{1}{\kappa^{D}}
$$

Now one should ask the exotic sliver to be a null element of the algebra

$$
\left|\hat{\Xi}_{\frac{\kappa+1}{\kappa}}\right\rangle^{(2)} *\left|\hat{\Xi}_{\epsilon}\right\rangle^{(2)}=\left|\hat{\Xi}_{\frac{\kappa+1}{\kappa}}\right\rangle^{(2)}
$$

which implies

$$
\mathcal{N}_{\epsilon}^{(2)}=\left(\frac{\epsilon}{\kappa+1}\right)^{D}
$$

In this case too the inverse algebra closes with structure constant 1 ,

$$
\left|\hat{\Xi}_{\epsilon}\right\rangle^{(2)} *\left|\hat{\Xi}_{\eta}\right\rangle^{(2)}=\left|\hat{\Xi}_{\epsilon \star \eta}\right\rangle^{(2)}
$$

Note that the dressed sliver has the same normalization and behaves as the identity in both algebras.

The next task is to compute the $b p z-$ norm of such states; here we limit ourselves to a formal expression, since all of them are constructed on the sliver which is known to have vanishing norm. This formal expression will be suitably regularized in the next section.

Using results from the appendix B, for states belonging to the (1) algebra we obtain

$$
\begin{aligned}
& { }^{(1)}\left\langle\hat{\Xi}_{\epsilon} \mid \hat{\Xi}_{\epsilon}\right\rangle^{(1)}=\left(\frac{\left(\mathcal{N}_{\epsilon}^{(1)}\right)^{2}}{\operatorname{det}\left(1-\hat{T}_{\epsilon}^{2}\right)^{\frac{1}{2}}} \operatorname{Det}(1-\mathcal{T} \mathcal{M})\right)^{D}\langle 0 \mid 0\rangle \\
& =\frac{V^{(D)}}{(2 \pi)^{D}}\left(\frac{(1+(1-\epsilon) \kappa)^{2}}{(1-\epsilon)(1+\kappa)(1+\kappa-\epsilon(\kappa-1))}\right)^{D}\left(\frac{\operatorname{Det}(1-\mathcal{T} \mathcal{M})}{\left(\operatorname{det}\left(1-T^{2}\right)\right)^{\frac{1}{2}}}\right)^{D}
\end{aligned}
$$


As we have just mentioned, this expression is formal, since, due to the fact that the third factor in the $r h s$ is vanishing, all norms in this algebra vanish as well, except perhaps for $\epsilon=1$ and $\epsilon=\frac{\kappa+1}{\kappa-1}$, for which the denominator of the second factor vanishes, and we get a $\frac{0}{0}$ expression.

A remark is in order concerning the state represented by $\epsilon=\frac{\kappa+1}{\kappa-1}$. This state is not a projector, but has the nice property of squaring to the dressed sliver, and can be therefore identified with a non trivial "square root" of unity

$$
\left|\hat{\Xi}_{\frac{\kappa+1}{\kappa-1}}\right\rangle^{(1)} *\left|\hat{\Xi}_{\frac{\kappa+1}{\kappa-1}}\right\rangle^{(1)}=\left|\hat{\Xi}_{1}\right\rangle^{(1)}
$$

It is quite natural therefore that, if the dressed sliver can have a finite norm, also its square root should. For what concerns the inverse algebra (2) all can be repeated with only slight modifications for the normalization factors $\mathcal{N}^{(2)}$, which never vanishes for states belonging to the algebra itself. Again we can have a non-vanishing norm for the dressed sliver and its square root, which has the same normalization as in the algebra (1). Therefore we will not repeat the computation of the norm. In any case, in the rest of the paper, we will deal only with the first algebra.

\section{The dressed sliver action: matter part.}

In the previous section we have introduced a Fock space state, depending on a parameter $\epsilon$, that interpolates between the sliver $\epsilon=0$ and the dressed sliver $\epsilon=1$. Now we intent to show that by its means, we can give a precise definition of the norm of the dressed sliver, so that both its norm and its action can be made finite.

As already mentioned above, the determinants in (2.19), (2.20) relevant to the sliver are ill-defined. They are actually well defined for any finite truncation of the matrix $X$ to level $L$ and need a regulator to account for its behaviour when $L \rightarrow \infty$. A regularization that fits particularly our needs was introduced by Okuyama [34 and we will use it here. It consists in using an asymptotic expression for the eigenvalue density $\rho(k)$ of $X$ (see also section 3), $\rho(k) \sim \frac{1}{2 \pi} \log L+\rho_{\text {fin }}(k)$, for large $L$, where $\rho_{\text {fin }}(k)$ is a finite contribution when $L \rightarrow \infty$, see [35]. This leads to asymptotic expressions for the various determinants we need. In particular the scale of $L$ can be chosen in such a way that

$$
\begin{aligned}
& \operatorname{det}(1+T)=h_{+} L^{-\frac{1}{3}}+\ldots \\
& \operatorname{det}(1-T)=h_{-} L^{\frac{1}{6}}+\ldots \\
& \operatorname{det}(1-X)=h_{X} L^{\frac{1}{9}}+\ldots
\end{aligned}
$$

where dots denote non-leading contribution when $L \rightarrow \infty$ and $h_{+}, h_{-}, h_{X}$ are suitable numerical constants which arise due to the finite contribution in the eigenvalue density ${ }^{4}$.

\footnotetext{
${ }^{4}$ In particular, for any infinite matrix $A$ which is diagonal in the $k$-basis, the determinant can be regularized by the level $L$ as

$$
\operatorname{det}(A)=h_{A} L^{\int_{-\infty}^{\infty} \frac{d k}{2 \pi} A(k)}, \quad h_{A}=e^{\int_{-\infty}^{\infty} d k \rho_{f i n}(k) A(k)} .
$$
}

We thank D.Belov for a discussion on this point. 
Our strategy consists in tuning $L$ with $\epsilon$ in such a way as to obtain finite results. Let us start, as a warm up exercise, with the $b p z$ norm $\left\langle\hat{\Xi}_{\epsilon} \mid \hat{\Xi}_{\epsilon}\right\rangle$. We have

$$
\left\langle\hat{\Xi}_{\epsilon} \mid \hat{\Xi}_{\epsilon}\right\rangle=\frac{\hat{\mathcal{N}}_{\epsilon}^{2}}{\left[\operatorname{det}\left(1-\hat{S}_{\epsilon}^{2}\right)\right]^{D / 2}}\langle 0 \| 0\rangle
$$

where $D=26$ and (see previous section)

$$
\hat{\mathcal{N}}_{\epsilon}=[\operatorname{Det}(1-\Sigma \mathcal{V})]^{D / 2} \mathcal{N}_{\epsilon}^{(1)}, \quad \mathcal{N}_{\epsilon}^{(1)}=\left(\frac{1+(1-\epsilon) \kappa}{\kappa+1}\right)^{D}
$$

Likewise we have

$$
\begin{aligned}
\operatorname{det}\left(1-\hat{S}_{\epsilon}^{2}\right) & =\operatorname{det}\left(1-\hat{T}_{\epsilon}^{2}\right)=\operatorname{det}\left(1-\hat{T}_{\epsilon}\right) \operatorname{det}\left(1+\hat{T}_{\epsilon}\right) \\
& =\operatorname{det}\left(1-T^{2}\right) \operatorname{det}\left(1-\epsilon P \frac{1}{1-T}\right) \operatorname{det}\left(1+\epsilon P \frac{1}{1+T}\right)
\end{aligned}
$$

Using the results of appendix B.3 we find

$$
\operatorname{det}\left(1-\hat{S}_{\epsilon}^{2}\right)=\operatorname{det}\left(1-T^{2}\right)(1-\epsilon)^{2}\left(\frac{\kappa+1-\epsilon(\kappa-1)}{\kappa+1}\right)^{2}
$$

Therefore, in the limit $\epsilon \rightarrow 1$ the dominant term will be

$$
\operatorname{det}\left(1-\hat{S}_{\epsilon}^{2}\right)=\operatorname{det}\left(1-T^{2}\right)(1-\epsilon)^{2}\left(\frac{2}{\kappa+1}\right)^{2}
$$

Now, recalling that $\operatorname{Det}(1-\Sigma \mathcal{V})=\operatorname{det}(1-X) \operatorname{det}(1+T)$, and putting together all the above results, we find

$$
\left\langle\hat{\Xi}_{\epsilon} \mid \hat{\Xi}_{\epsilon}\right\rangle=\left(h \frac{1}{4(\kappa+1)^{2}} \frac{L^{-\frac{5}{18}}}{(1-\epsilon)^{2}}+\ldots\right)^{\frac{D}{2}}\langle 0 \mid 0\rangle, \quad h=\frac{h_{X}^{2} h_{+}}{h_{-}}
$$

where dots denote irrelevant terms in the limit $\epsilon \rightarrow 1$ and $L \rightarrow \infty$. Therefore, if we assume that

$$
1-\epsilon=s L^{-\frac{5}{36}}
$$

for some constant $s$, we have

$$
\lim _{\epsilon \rightarrow 1}\left\langle\hat{\Xi}_{\epsilon} \mid \hat{\Xi}_{\epsilon}\right\rangle=\left(\frac{h}{4(\kappa+1)^{2} s^{2}}\right)^{\frac{D}{2}}\langle 0 \mid 0\rangle
$$

which may take any prescribed positive finite value ${ }^{5}$. The factor $\langle 0 \mid 0\rangle=\delta^{(D)}(0)$ is normalized to $\frac{V^{(D)}}{(2 \pi)^{D}}$. We notice for later use that, in order for such prescription to be consistent, it must be that if we rescale $1-\epsilon, L^{-\frac{5}{36}}$ should be accordingly rescaled so that their ratio is always $s$. This is in order to guarantee that the limit be scale independent.

It would look natural to define the number $(5.9)$ as the norm $\langle\hat{\Xi} \mid \hat{\Xi}\rangle$ of our regularized dressed sliver. However, as we shall see next, the regularization prescription defined by

\footnotetext{
${ }^{5}$ It is obvious that the constants $\kappa+1$ and $h$ could be absorbed in $s$.
} 
eqs. 5.7, 5.8,5.9) does not guarantee that the equations of motion be satisfied in the action. In fact, as it turns out (see below),

$$
\lim _{\epsilon \rightarrow 1}\left\langle\hat{\Xi}_{\epsilon} \mid \hat{\Xi}_{\epsilon}\right\rangle \neq \lim _{\epsilon \rightarrow 1}\left\langle\hat{\Xi}_{\epsilon} \mid \Xi_{\epsilon} * \hat{\Xi}_{\epsilon}\right\rangle
$$

There is here a subtle problem. We delve into it by analyzing the quantity

$$
\left\langle\hat{\Xi}_{\epsilon_{1}} \mid \hat{\Xi}_{\epsilon_{2}}\right\rangle=\frac{\hat{\mathcal{N}}_{\epsilon_{1}} \hat{\mathcal{N}}_{\epsilon_{2}}}{\operatorname{det}\left(1-\hat{S}_{\epsilon_{1}} \hat{S}_{\epsilon_{2}}\right)}\langle 0 \mid 0\rangle
$$

The analysis carried out in Appendix C leads us to infinite many ways of taking the limit $\epsilon_{1}, \epsilon_{2} \rightarrow 1$, with results that vary in a finite range. At one extreme we have the result obtained above, which corresponds to $\epsilon_{1}=\epsilon_{2}=\epsilon$. At the the other extreme we have the ordered limit

$$
\lim _{\epsilon_{1} \rightarrow 1}\left(\lim _{\epsilon_{2} \rightarrow 1}\left\langle\hat{\Xi}_{\epsilon_{1}} \mid \hat{\Xi}_{\epsilon_{2}}\right\rangle\right)
$$

According to Appendix $\mathrm{C}$, when $\epsilon_{1}$ and $\epsilon_{2}$ are in the vicinity of 1 we have

$$
\begin{aligned}
& \frac{1}{\langle 0 \mid 0\rangle}\left\langle\hat{\Xi}_{\epsilon_{1}} \mid \hat{\Xi}_{\epsilon_{2}}\right\rangle \\
& =\left(\frac{\operatorname{Det}(1-\Sigma \mathcal{V})}{\sqrt{\operatorname{det}\left(1-S^{2}\right)}}\right)^{D}\left(\frac{1}{4(\kappa+1)^{2}}\right)^{\frac{D}{2}}\left(\frac{4}{\left(\kappa\left(1-\epsilon_{1}\right)\left(1-\epsilon_{2}\right)+1-\epsilon_{1} \epsilon_{2}\right)^{2}}\right)^{\frac{D}{2}}+\ldots
\end{aligned}
$$

where dots denote non-leading terms. Now let as take the limit (5.12)

$$
\begin{aligned}
& \frac{1}{\langle 0 \mid 0\rangle} \lim _{\epsilon_{1} \rightarrow 1}\left(\lim _{\epsilon_{2} \rightarrow 1}\left\langle\hat{\Xi}_{\epsilon_{1}} \mid \hat{\Xi}_{\epsilon_{2}}\right\rangle\right) \\
& =\lim _{\epsilon_{1} \rightarrow 1}\left(\frac{\operatorname{Det}(1-\Sigma \mathcal{V})}{\sqrt{\operatorname{det}\left(1-S^{2}\right)}}\right)^{D}\left(\frac{1}{4(\kappa+1)^{2}}\right)^{\frac{D}{2}}\left(\frac{4}{\left(1-\epsilon_{1}\right)^{2}}\right)^{\frac{D}{2}}+\ldots \\
& =\lim _{\epsilon_{1} \rightarrow 1}\left(\frac{h}{(\kappa+1)^{2}}\right)^{\frac{D}{2}}\left(\frac{L^{-\frac{5}{36}}}{1-\epsilon_{1}}\right)^{D}+\ldots=\left(\frac{h}{(\kappa+1)^{2} s_{1}^{2}}\right)^{\frac{D}{2}}
\end{aligned}
$$

provided

$$
1-\epsilon_{1}=s_{1} L^{-\frac{5}{36}}
$$

It is easy to see that if we reverse the order of the limits in (5.12) we obtain the same result.

Between this result and (5.9) there is a discrepancy, a factor of 4 . This factor can be absorbed into a redefinition of $s, s_{1}=2 s$. Had we adopted still another method of taking the limit we would have obtained a result in between. In conclusion there are infinite many ways of deriving the norm starting from $\left\langle\hat{\Xi}_{\epsilon_{1}} \mid \hat{\Xi}_{\epsilon_{2}}\right\rangle$, they all lead to the same result up to a redefinition of the $s$ factor.

Now the question is: do we have a criterion to select among all these different limits? The answer is: yes, we do. It is the requirement that the equation of motion be satisfied, i.e. we must have

$$
\lim _{\epsilon_{1}, \epsilon_{2} \rightarrow 1}\left\langle\hat{\Xi}_{\epsilon_{1}} \mid \hat{\Xi}_{\epsilon_{2}}\right\rangle=\lim _{\epsilon_{1}, \epsilon_{2}, \epsilon_{3} \rightarrow 1}\left\langle\hat{\Xi}_{\epsilon_{1}} \mid \hat{\Xi}_{\epsilon_{2}} * \hat{\Xi}_{\epsilon_{3}}\right\rangle
$$


The analysis carried out in Appendix $\mathrm{C}$ of the expression in the rhs tells us that once again there are infinite many ways to calculate the triple limit, and there are infinite many ways to satisfy (5.15). For instance, the limit $\epsilon_{1}=\epsilon_{2}=\epsilon_{3} \rightarrow 1$ does not satisfy (5.15), while the criterion of the ordered limits does, i.e. that

$$
\lim _{\epsilon_{1} \rightarrow 1}\left(\lim _{\epsilon_{2} \rightarrow 1}\left\langle\hat{\Xi}_{\epsilon_{1}} \mid \hat{\Xi}_{\epsilon_{2}}\right\rangle\right)=\lim _{\epsilon_{1} \rightarrow 1}\left(\lim _{\epsilon_{2} \rightarrow 1}\left(\lim _{\epsilon_{3} \rightarrow 1}\left\langle\hat{\Xi}_{\epsilon_{1}} \mid \hat{\Xi}_{\epsilon_{2}} * \hat{\Xi}_{\epsilon_{3}}\right\rangle\right)\right)
$$

First we notice that due to the symmetry of $\left\langle\hat{\Xi}_{\epsilon_{1}} \mid \hat{\Xi}_{\epsilon_{2}} * \hat{\Xi}_{\epsilon_{3}}\right\rangle$ (see Appendix C2), the order $1,2,3$ in the last limit is irrelevant. What is relevant is that the limits are taken in succession. Now, using the formulas of the previous section and of Appendix B, it is easy to see that

$$
\lim _{\epsilon_{3} \rightarrow 1}\left\langle\hat{\Xi}_{\epsilon_{1}} \mid \hat{\Xi}_{\epsilon_{2}} * \hat{\Xi}_{\epsilon_{3}}\right\rangle=\lim _{\epsilon_{3} \rightarrow 1}\left\langle\hat{\Xi}_{\epsilon_{1}} \mid \hat{\Xi}_{\epsilon_{2} \star \epsilon_{3}}\right\rangle=\left\langle\hat{\Xi}_{\epsilon_{1}} \mid \hat{\Xi}_{\epsilon_{2}}\right\rangle
$$

Therefore (5.15) follows.

As we mentioned before, there are other ways of taking the limit $\epsilon_{i} \rightarrow 1$ while satisfying (5.15). However the ordered limits seem to have a privileged status, as we will try to explain next. We would like to show that the equation of motion (2.6) holds in a more general sense than eq.(5.16). In other words we would like that

$$
\lim _{\epsilon_{1} \rightarrow 1}\left\langle\Psi \mid \hat{\Xi}_{\epsilon_{1}}\right\rangle=\lim _{\epsilon_{1}, \epsilon_{2} \rightarrow 1}\left\langle\Psi \mid \hat{\Xi}_{\epsilon_{1}} * \hat{\Xi}_{\epsilon_{2}}\right\rangle
$$

for 'any' state $\Psi$. In order to appreciate the problem one should recall that the limiting procedure is necessary whenever evaluations of determinants are involved, otherwise it is irrelevant. Therefore if $\Psi$ is any state of the Fock space constructed by applying to the vacuum a polynomial of the string creation operators, eq. 5.18) holds; the only proviso is that, since $\hat{\Xi}_{\epsilon}$ contains a normalization which vanishes when $L \rightarrow \infty$ (but it diverges in the ghost case, see below), we must take this limit as the last operation.

The validity of eq.(5.18) may be in danger only when $\Psi$ is a close relative to $\hat{\Xi}$. We have already seen how to deal with the case $\hat{\Xi}_{\epsilon}$. The conclusion does not change if the $\hat{\Xi}_{\epsilon}$ is multiplied by a polynomial of the string creation operators or even by a coherent state contructed out of the latter. One may ask what happens when $\Psi$ coincides with $\hat{\Xi}$ itself. In this case the expressions under the limit symbols in eq.(5.18) make sense, and we have to make sure that the equation holds. It is easy to see that, once again, it holds with the ordered limiting procedure. The set of states $\Psi$ for which (5.18) holds, does not exhaust all the states one can think of, however it contains all Fock space states as well as all the states that are relevant to this and the companion paper, [41]. To characterize these limitiations we say that the EOM holds in a weak sense.

From now on we assume the ordered limit procedure as the good limiting procedure. In particular the norm of $\hat{\Xi}$ is defined by eq.(5.13).

What we have achieved so far is to prove that it is possible to assign a finite positive number to the expression (norm) $\langle\hat{\Xi} \mid \hat{\Xi}\rangle$, in a way which is consistent with the matter equation of motion. It does not mean that a state exists in the Hilbert space which is the limit of $\hat{\Xi}_{\epsilon}$ when $\epsilon \rightarrow 1$. In order to show this one would have to prove that the number 
$\left\|\hat{\Xi}_{\epsilon_{1}}-\hat{\Xi}_{\epsilon_{2}}\right\|^{2}$ becomes smaller and smaller when $\epsilon_{1}, \epsilon_{2} \rightarrow 1$. In such a case Hilbert space completeness would guarantee the existence of a limiting state. Now

$$
\left\|\hat{\Xi}_{\epsilon_{1}}-\hat{\Xi}_{\epsilon_{2}}\right\|^{2}=\left\langle\hat{\Xi}_{\epsilon_{1}} \mid \hat{\Xi}_{\epsilon_{1}}\right\rangle+\left\langle\hat{\Xi}_{\epsilon_{2}} \mid \hat{\Xi}_{\epsilon_{2}}\right\rangle-2\left\langle\hat{\Xi}_{\epsilon_{1}} \mid \hat{\Xi}_{\epsilon_{2}}\right\rangle
$$

The first two terms are similar to (5.2), while the last term has been calculated in (C.4). From the latter equation it is evident that the Cauchy condition would be satisfied if the term in the expression in the second line of eq. C.4 were to approach 1 when $\epsilon_{1}, \epsilon_{2} \rightarrow 1$. However, as seen in Appendix C1, this quantity remains at a finite distance from 1 when $\epsilon_{1}, \epsilon_{2} \rightarrow 1$, unless one takes $\epsilon_{1}=\epsilon_{2}$. The conclusion is that we cannot satisfy the Cauchy condition for the sequence $\hat{\Xi}_{\epsilon}$.

Therefore, while the regularization procedure defined above guarantees that we can associate a positive finite number to the symbol $\langle\hat{\Xi} \mid \hat{\Xi}\rangle$, it does not allow us to associate any Hilbert space state to $\hat{\Xi}$. The state $\hat{\Xi}$ lives outside the Hilbert space. A careful treatment of this problem would require embedding the string theory Hilbert space into a larger space with suitably defined topology, according to which $\lim _{\epsilon \rightarrow 1} \hat{\Xi}_{\epsilon}=\hat{\Xi}$ makes full sense. This interesting issue goes beyond the scope of the present paper.

\section{The ghost dressed sliver}

In this section our purpose is to find the ghost companion of the regularized dressed sliver solution discussed above. The previous analysis for the matter part can be easily extended also to the ghost part.

Let us start with the definition of the $*_{g}$ product:

$$
|\widetilde{\Psi}\rangle *_{g}|\widetilde{\Phi}\rangle={ }_{1}\left\langle\left.\widetilde{\Psi}\right|_{2}\left\langle\widetilde{\Phi} \mid \widetilde{V}_{3}\right\rangle\right.
$$

where the ghost part of the 3 -strings vertex is defined by

$$
\left|\widetilde{V}_{3}\right\rangle=\exp \left[\sum_{r, s=1}^{3}\left(\sum_{n, m=1}^{\infty} c_{n}^{(r) \dagger} \widetilde{V}_{n m}^{r s} b_{m}^{(s) \dagger}+\sum_{n=1}^{\infty} c_{n}^{(r) \dagger} \widetilde{V}_{n 0}^{r s} b_{0}^{(s)}\right)\right] \prod_{r=1}^{3}\left(c_{0}^{(r)} c_{1}^{(r)}\right)|0\rangle_{123}
$$

Here $c_{n}^{(r)}$ and $b_{n}^{(r)}$ are the standard ghost oscillator modes of the $r$-th string, which satisfy

$$
\left\{b_{n}^{(r)}, c_{m}^{(s) \dagger}\right\}=\delta_{n m} \delta_{r s}, \quad b_{n}^{(r) \dagger}=b_{-n}^{(r)}, \quad c_{n}^{(r) \dagger}=c_{-n}^{(r)}
$$

and $|0\rangle_{123} \equiv|0\rangle_{1} \otimes|0\rangle_{2} \otimes|0\rangle_{3}$ is the tensor product of the $\mathrm{SL}(2, \mathbf{R})$-invariant ghost vacuum states, normalized such that

$$
\left\langle 0\left|c_{1}^{\dagger} c_{0} c_{1}\right| 0\right\rangle=1
$$

The symbols $\widetilde{V}_{n m}^{r s}$ and $\widetilde{V}_{n 0}^{r s}$ are coefficients computed in [25, 26, 27, 30] and their properties necessary for us here ${ }^{6}$ are listed in appendix A. The $b p z$ conjugation properties are

$$
\text { bpz }\left(c_{n}^{(r)}\right)=(-1)^{n+1} c_{-n}^{(r)}, \quad b p z\left(b_{n}^{(r)}\right)=(-1)^{n} b_{-n}^{(r)} .
$$

\footnotetext{
${ }^{6}$ For a more complete discussion see [27, 33].
} 
It was shown in [13] that there is a simple solution of the ghost field equation (2.5) in the form of the squeezed state

$$
|\widetilde{\Xi}\rangle=\widetilde{\mathcal{N}} \exp \left(\sum_{n, m=1}^{\infty} c_{n}^{\dagger} \widetilde{S}_{n m} b_{m}^{\dagger}\right) c_{1}|0\rangle,
$$

where the matrix $\widetilde{S}$ satisfies the equation

$$
\widetilde{S}=\widetilde{V}^{11}+\left(\widetilde{V}^{12}, \widetilde{V}^{21}\right)(I-\widetilde{\Sigma} \widetilde{\mathcal{V}})^{-1} \widetilde{\Sigma}\left(\begin{array}{c}
\widetilde{V}^{21} \\
\widetilde{V}^{12}
\end{array}\right)
$$

which has exactly the same form as (2.15) $(\widetilde{\Sigma}$ and $\widetilde{\mathcal{V}}$ are defined as in (2.16)), but now with tildes. As before one introduces $\widetilde{X}=C \widetilde{V}^{11}, \widetilde{X}_{+}=C \widetilde{V}^{12}$ and $\widetilde{X}_{-}=C \widetilde{V}^{21}$ (see appendix A for properties). As the $\tilde{X}_{i}$ 's satisfy the same algebraic relatons as the $X_{i}$ 's, one can construct solutions to (6.4) the same way as for the matter part. The solution we are interested in, in terms of $\widetilde{T}=C \widetilde{S}$, is

$$
\widetilde{T}=\frac{1}{2 \widetilde{X}}(1+\widetilde{X}-\sqrt{(1+3 \widetilde{X})(1-\widetilde{X})})
$$

The normalization constant is

$$
\widetilde{\mathcal{N}}=-[\operatorname{Det}(1-\tilde{\Sigma} \tilde{\mathcal{V}})]^{-1}
$$

The contribution of the ghost part to the action is given by

$$
\langle\widetilde{\Xi}|\mathcal{Q}| \widetilde{\Xi}\rangle=\widetilde{\mathcal{N}}^{2} \operatorname{det}\left(1-\widetilde{S}^{2}\right)
$$

Now the determinants in eqs. (6.5) and (6.6) are both vanishing, in such a way that the ghost part of the action diverges (see below). When one combines this with the results for the matter part (using (2.4), (2.19) and (2.20)) one finds 34 that both normalization constant and action of the overall state vanish.

Following the analysis of the matter part, we consider deformations of this solution. We introduce two real vectors $\beta=\left\{\beta_{n}\right\}$ and $\delta=\left\{\delta_{n}\right\}$ which satisfy

$$
\widetilde{\rho}_{1} \beta=\widetilde{\rho}_{1} \delta=0, \quad \widetilde{\rho}_{2} \beta=\beta, \quad \widetilde{\rho}_{2} \delta=\delta .
$$

We also set

$$
\left\langle\beta\left|\frac{1}{1-\widetilde{T}^{2}}\right| \delta\right\rangle=1, \quad\left\langle\beta\left|\frac{\widetilde{T}}{1-\widetilde{T}^{2}}\right| \delta\right\rangle=\widetilde{\kappa}
$$

where the first equation fixes the relative normalization of $\beta$ and $\delta$, and the second defines $\widetilde{\kappa}$. Note that one can repeat the analysis of section 3: since the eigenvalues of the ghost sliver matrix $\widetilde{T}$ are the opposite of the eigenvalues of the corresponding matter matrix $T$, it follows that $\widetilde{\kappa}$ is non-negative.

We now dress the ghost part of the sliver and introduce the squeezed state

$$
\left|\widehat{\widetilde{\Xi}}_{\tilde{\epsilon}}\right\rangle=\widehat{\widetilde{\mathcal{N}}}_{\tilde{\epsilon}} e^{c^{\dagger} \widehat{\widetilde{S}}_{\tilde{\epsilon}} b^{\dagger}} c_{1}|0\rangle
$$


where instead of $\widetilde{S}$ we now have

$$
\widehat{\widetilde{S}}_{\tilde{\epsilon}}=\widetilde{S}+\tilde{\epsilon} \widetilde{R}, \quad \widetilde{R}=\frac{1}{\widetilde{\kappa}+1}(|C \delta\rangle\langle\beta|+| \delta\rangle\langle C \beta|)
$$

It it easy to see that $\widehat{\widetilde{S}}_{\tilde{\epsilon}}^{*}=C \widehat{\widetilde{S}}_{\tilde{\epsilon}} C$ for $\beta, \delta$ real, which means that the string field is real.

Let us now calculate $\widehat{\widetilde{\Xi}}_{\tilde{\epsilon} *_{g}} \widehat{\widetilde{\Xi}}_{\tilde{\eta}}$, where both states have the same $\beta$ and $\delta$. If one defines, the reduced $*_{b_{0}}$-product as, [32],

$$
\widehat{\widetilde{\Xi}}_{\tilde{\epsilon}} * b_{0} \widehat{\widetilde{\Xi}}_{\tilde{\eta}} \equiv b_{0}\left(\widetilde{\Xi}_{\tilde{\epsilon}} * g \widehat{\widetilde{\Xi}}_{\tilde{\eta}}\right),
$$

then one can immediately see that it can be calculated using the vertex (6.2) but without terms containing $b_{0}$ modes (reduced vertex). Then the calculation of the reduced product (6.10) repeats essentially the calculation in the matter sector of sec. 4 , the only differences being that untilded objects are replaced by the corresponding tilded ones and, more important, the determinants are raised to the power $-2 / D$ with respect to the corresponding matter ones (this is because of the anticommutativity of ghosts). The result is then

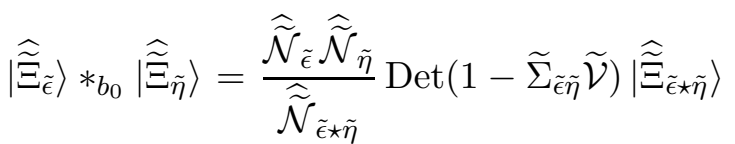

$$
\begin{aligned}
& =\frac{\widehat{\widetilde{\mathcal{N}}}_{\tilde{\epsilon}} \widehat{\widetilde{\mathcal{N}}}_{\tilde{\eta}}}{\widehat{\widetilde{\mathcal{N}}}_{\tilde{\epsilon} \star \tilde{\eta}}}\left[\frac{1+(1-\tilde{\epsilon})(1-\tilde{\eta}) \widetilde{\kappa}}{\widetilde{\kappa}+1}\right]^{2} \operatorname{Det}(1-\widetilde{\Sigma} \widetilde{\mathcal{V}})\left|\widehat{\widetilde{\Xi}}_{\tilde{\epsilon} \star \tilde{\eta}}\right\rangle,
\end{aligned}
$$

where the $\star$ multiplication rule is defined in 4.9 ).

Now, it was shown in [32] that, if states $A$ and $B$ are in the subspace spanned by coherent states, then the $*_{g}$ product can be obtained from the reduced product using

$$
A *_{g} B=\mathcal{Q}\left(A *_{b_{0}} B\right)
$$

which applied to (6.11) gives

$$
\left|\widehat{\widetilde{\Xi}}_{\tilde{\epsilon}}\right\rangle *_{g}\left|\widehat{\widetilde{\Xi}}_{\tilde{\eta}}\right\rangle=\frac{\widehat{\widetilde{\mathcal{N}}}_{\tilde{\epsilon}} \widehat{\widetilde{\mathcal{N}}}_{\tilde{\eta}}}{\widehat{\widetilde{\mathcal{N}}}_{\tilde{\epsilon} \star \tilde{\eta}}}\left[\frac{1+(1-\tilde{\epsilon})(1-\tilde{\eta}) \widetilde{\kappa}}{\widetilde{\kappa}+1}\right]^{2} \operatorname{Det}(1-\widetilde{\Sigma} \widetilde{\mathcal{V}}) \mathcal{Q}\left|\widehat{\widetilde{\Xi}}_{\tilde{\epsilon} \star \tilde{\eta}}\right\rangle .
$$

However, a more careful derivation is needed because states like (6.9) are (at least apparently) not of the required form and it could be risky to use the above argument. In appendix D we give a direct proof that 6.13 is correct.

At this point one should observe a formal similarity between eq. (6.13) and the corresponding one in the matter sector (4.13). In fact, one can now basically repeat the arguments of sections 4 and 5 with only minor modifications.

First, it is natural to choose a normalization such that (6.13) has the following form

$$
\left|\widehat{\widetilde{\Xi}}_{\tilde{\epsilon}}\right\rangle *_{g}\left|\widehat{\widetilde{\Xi}}_{\tilde{\eta}}\right\rangle=-\mathcal{Q}\left|\widehat{\widetilde{\Xi}}_{\tilde{\epsilon} \star \tilde{\eta}}\right\rangle
$$


Again, there are two different normalizations with this property, given by

$$
\begin{aligned}
& \widehat{\widetilde{\mathcal{N}}}_{\tilde{\epsilon}}^{(1)}=-\left(\frac{\widetilde{\kappa}+1}{1+(1-\tilde{\epsilon}) \widetilde{\kappa}}\right)^{2}[\operatorname{Det}(1-\tilde{\Sigma} \widetilde{\mathcal{V}})]^{-1} \\
& \widehat{\widetilde{\mathcal{N}}}_{\tilde{\epsilon}}^{(2)}=-\left(\frac{\widetilde{\kappa}+1}{\tilde{\epsilon}}\right)^{2}[\operatorname{Det}(1-\widetilde{\Sigma} \widetilde{\mathcal{V}})]^{-1} .
\end{aligned}
$$

The first one is singular in $\tilde{\epsilon}=\frac{\tilde{\kappa}+1}{\tilde{\kappa}}$, and the second one in $\tilde{\epsilon}=0$. From now on we use exclusively the first one, and drop the ${ }^{(1)}$ in superscript.

From (6.14) it follows that our states (6.9) satisfy the ghost equation of motion when

$$
\tilde{\epsilon} \star \tilde{\epsilon}=\tilde{\epsilon}
$$

and we already know that it is true only for

$$
\tilde{\epsilon}=0,1, \frac{\widetilde{\kappa}+1}{\widetilde{\kappa}}
$$

Again, beside the Hata-Kawano solution (i.e. the solution with $\tilde{\epsilon}=0$ ), we obtain in addition two families of solutions, depending on the choice of $\beta$ and $\delta$.

Now we show that for the solution with $\tilde{\epsilon} \rightarrow 1$ (ghost part of the dressed sliver) we can define a finite action. We shall consider first the kinetic term, for which we need

$$
\begin{aligned}
\left\langle\widehat{\widetilde{\Xi}}_{\tilde{\epsilon}_{1}}|\mathcal{Q}| \widehat{\widetilde{\Xi}}_{\tilde{\epsilon}_{2}}\right\rangle & =\left\langle\widehat{\widetilde{\Xi}}_{\tilde{\epsilon}_{1}}\left|c_{0}\right| \widehat{\widetilde{\Xi}}_{\tilde{\epsilon}_{2}}\right\rangle=\widehat{\widetilde{\mathcal{N}}}_{\tilde{\epsilon}_{1}} \widehat{\widetilde{\mathcal{N}}}_{\tilde{\epsilon}_{2}} \operatorname{det}\left(1-\widehat{\widetilde{S}}_{\tilde{\epsilon}_{1}} \widehat{\widetilde{S}}_{\tilde{\epsilon}_{2}}\right) \\
& =\left(1-\prod_{i=1}^{2} \frac{\tilde{\epsilon}_{i}}{1+\left(1-\tilde{\epsilon}_{i}\right) \tilde{\kappa}}\right)^{2} \frac{\operatorname{det}(1-\widetilde{\widetilde{S}})}{[\operatorname{Det}(1-\widetilde{\Sigma} \widetilde{\mathcal{V}})]^{2}} .
\end{aligned}
$$

where in the last line we used $(6.15)$ and $(B .35)$. It was shown in 34 that the level truncation regularization at the leading order leads to

$$
\frac{\operatorname{det}\left(1-\widehat{\widetilde{S}}^{2}\right)}{[\operatorname{Det}(1-\tilde{\Sigma} \tilde{\mathcal{V}})]^{2}}=\frac{L^{\frac{11}{18}}}{\tilde{h}}+\ldots
$$

where $\tilde{h}$ is the numerical factor analogous to $h$ for the ghost part. The $r h s$ of $(6.20)$ diverges when the cutoff is lifted, i.e., when $L \rightarrow \infty$. But, as for the matter part, we see that if we let $\tilde{\epsilon} \rightarrow 1$ in a specific way, the expression (6.19) can be made finite. Following our discussion from sec. 5 we use the ordered limits procedure, which, using (6.20), gives

$$
\begin{aligned}
\lim _{\tilde{\epsilon}_{1} \rightarrow 1}\left(\lim _{\tilde{\epsilon}_{2} \rightarrow 1}\left\langle\widehat{\widetilde{\Xi}}_{\tilde{\epsilon}_{1}}|\mathcal{Q}| \widehat{\widetilde{\Xi}}_{\tilde{\epsilon}_{2}}\right\rangle\right) & =\lim _{\tilde{\epsilon}_{1} \rightarrow 1}\left[1-\frac{\tilde{\epsilon}_{1}}{1+\left(1-\tilde{\epsilon}_{1}\right) \tilde{\kappa}}\right]^{2} \frac{\operatorname{det}\left(1-\widehat{\widetilde{S}}^{2}\right)}{[\operatorname{Det}(1-\tilde{\Sigma} \tilde{\mathcal{V}})]^{2}} \\
& =\lim _{\tilde{\epsilon}_{1} \rightarrow 1}\left[\frac{(\tilde{\kappa}+1)\left(1-\tilde{\epsilon}_{1}\right)}{1+\left(1-\tilde{\epsilon}_{1}\right) \tilde{\kappa}}\right]^{2} \frac{L^{\frac{11}{18}}}{\tilde{h}}+\ldots .
\end{aligned}
$$

Therefore, if we assume that

$$
1-\tilde{\epsilon}_{1}=\tilde{s} L^{-\frac{11}{36}}
$$


for some constant $\tilde{s}$, we have

$$
\lim _{\tilde{\epsilon}_{1} \rightarrow 1}\left(\lim _{\tilde{\epsilon}_{2} \rightarrow 1}\left\langle\widehat{\widetilde{\Xi}}_{\tilde{\epsilon}_{1}}|\mathcal{Q}| \widehat{\widetilde{\Xi}}_{\tilde{\epsilon}_{2}}\right\rangle\right)=(\widetilde{\kappa}+1)^{2} \frac{\tilde{s}^{2}}{\tilde{h}} .
$$

which defines a finite value for the kinetic term in the action.

The calculation for the cubic term in the action goes along similar lines. One obtains here too that the ordered limits preserve the equation of motion,

$$
\lim _{\tilde{\epsilon}_{1} \rightarrow 1}\left(\lim _{\tilde{\epsilon}_{2} \rightarrow 1}\left(\lim _{\tilde{\epsilon}_{3} \rightarrow 1}\left\langle\widehat{\widetilde{\Xi}}_{\tilde{\epsilon}_{1}} \mid \widehat{\widetilde{\Xi}}_{\tilde{\epsilon}_{2}} * g \widehat{\widetilde{\Xi}}_{\tilde{\epsilon}_{3}}\right\rangle\right)\right)=-\lim _{\tilde{\epsilon}_{1} \rightarrow 1}\left(\lim _{\tilde{\epsilon}_{2} \rightarrow 1}\left\langle\widehat{\widetilde{\Xi}}_{\tilde{\epsilon}_{1}}|\mathcal{Q}| \widehat{\widetilde{\Xi}}_{\tilde{\epsilon}_{2}}\right\rangle\right)
$$

It is worth noting that, using the results of [33], the ghost companion of the dressed sliver can be easily shown to be (proportional to) a projector of the $b c$-twisted $*$-product [10].

\subsection{Overall regularized action}

Now we are ready to draw the conclusion concerning the regularized action. We collect the results (5.13,6.23) and plug them into (2.7). The action of the regularized dressed sliver is

$$
-\frac{\mathcal{S}(\hat{\Psi})}{V^{(D)}}=\frac{1}{6 g_{0}^{2}(2 \pi)^{D}} \frac{(\tilde{\kappa}+1)^{2} \tilde{s}^{2}}{(\kappa+1)^{D} s^{D}} \frac{h^{\frac{D}{2}}}{\tilde{h}}
$$

The value of the rhs can now be tuned to the physical value of the D25-brane tension. We stress that, apart from $g_{0}$, the parameters in the rhs are not present in the initial action, but arise from the regularization procedure ${ }^{7}$. More comments on this point can be found in section 8.

We would also like to point out that the regularized action (6.25) is not the only possibility. We could, for instance, connect in various ways the ghost and matter asymptotic expansions, to get an overall finite action. We could perhaps use also the limits $\kappa, \tilde{\kappa} \rightarrow-1$. At this stage we cannot decide what the best prescription is. Hopefully the study of the spectrum will shed light on this problem.

\section{Other finite norm solutions.}

In this section we discuss a few further issues concerning dressed slivers, without going into detailed calculations.

- Multiply dressed slivers. The most obvious generalization of the dressed sliver definition (2.23) consists in adding to $\hat{S}$ another operator $R^{\prime}$ with the same structure as $R$ and $\xi$ replaced by $\xi^{\prime}$, with

$$
\rho_{1} \xi^{\prime}=0, \quad \rho_{2} \xi^{\prime}=\xi^{\prime}
$$

and

$$
\xi^{\prime T} \frac{1}{1-T^{2}} \xi^{\prime}=1, \quad \xi^{\prime T} \frac{T}{1-T^{2}} \xi^{\prime}=\kappa^{\prime}
$$

\footnotetext{
${ }^{7}$ We remark that $\kappa, \tilde{\kappa}, h, \tilde{h}$ could be reabsorbed in the free parameters $s, \tilde{s}$.
} 
the components of $\xi^{\prime}$ being real and $\kappa^{\prime}$ a real number. The matrix $\hat{T}$ will be replaced by

$$
\hat{T}^{\prime}=T+P+P^{\prime}, \quad P^{\prime}=\frac{1}{\kappa^{\prime}+1}\left(\left|\xi^{\prime}\right\rangle\left\langle\xi^{\prime}|+| C \xi^{\prime}\right\rangle\left\langle C \xi^{\prime}\right|\right)
$$

The obvious question is whether this new state is a projector. In general it is not, but if $\xi^{\prime}$ satisfies the 'orthogonality' conditions

$$
\xi^{T} \frac{1}{1-T^{2}} \xi^{\prime}=0, \quad \xi^{T} \frac{T}{1-T^{2}} \xi^{\prime}=0
$$

then it is easy to repeat the proof of section 2 and conclude that the squeezed state with structure matrix $\hat{S}^{\prime}=S+R+R^{\prime}$ is in fact a projector. On the basis of section 3, one can see that the conditions (7.4) are easy to implement.

Again, the norm (and the action) of this new projector is ill-defined. We can introduce deformation parameters $\epsilon$ before $P$ and $\epsilon^{\prime}$ before $P^{\prime}$, and repeat what we did in section 3, 4 and 5 . For instance, for $\epsilon, \epsilon^{\prime}$ near 1 , denoting by $\hat{S}_{\epsilon, \epsilon^{\prime}}$ the relevant Neumann matrix,

$$
\begin{aligned}
& \operatorname{det}\left(1-\hat{S}_{\epsilon, \epsilon^{\prime}}^{2}\right)=\operatorname{det}\left(1-T^{2}\right)(1-\epsilon)^{2}\left(1-\epsilon^{\prime}\right)^{2} \frac{16}{(\kappa+1)^{2}\left(\kappa^{\prime}+1\right)^{2}} \\
& \operatorname{Det}\left(1-\hat{\Sigma}_{\epsilon, \epsilon^{\prime}}^{\prime} \mathcal{V}\right)=\operatorname{Det}(1-\mathcal{T} \mathcal{M}) \frac{1}{(\kappa+1)^{2}\left(\kappa^{\prime}+1\right)^{2}}
\end{aligned}
$$

and so on. It is obvious that we can add to $\hat{S}$ as many perturbations as we wish and still get projectors. For instance, if we add $R^{\prime \prime}$, specified by $\xi^{\prime \prime}$, with the same properties as $\xi$, the only condition we have to impose is that $\xi^{\prime \prime}$ be orthogonal to both $\xi$ and $\xi^{\prime}$ in the sense of eq.(7.4).

- Other projectors. Starting from the dressed sliver solutions it is rather easy to construct many others which are $*$-orthogonal to the dressed sliver, according to the construction initiated in [8] and fully implemented in [38]. First we introduce a real vector $\zeta^{\mu}=\left\{\zeta_{n}^{\mu}\right\}$ (notice the Lorentz index!), which is chosen to satisfy the conditions

$$
\rho_{1} \zeta^{\mu}=0, \quad \rho_{2} \zeta^{\mu}=\zeta^{\mu}, \quad \forall \mu
$$

and

$$
\left\langle\zeta^{\mu}\left|\frac{1}{1-T^{2}}\right| \zeta^{\nu}\right\rangle \eta_{\mu \nu}=1, \quad\left\langle\zeta^{\mu}\left|\frac{T}{1-T^{2}}\right| \zeta^{\nu}\right\rangle \eta_{\mu \nu}=\lambda
$$

Next we set

$$
\mathrm{x}=-\left(a^{\mu \dagger} \zeta^{\nu} \eta_{\mu \nu}\right)\left(a^{\mu \dagger} C \zeta^{\nu} \eta_{\mu \nu}\right)
$$

introduce the Laguerre polynomials $L_{n}(\mathrm{x} / \lambda)$ and define the states $\left|\hat{\Lambda}_{n}\right\rangle$ as follows

$$
\left|\hat{\Lambda}_{n}\right\rangle=(-\lambda)^{n} L_{n}\left(\frac{\mathrm{X}}{\lambda}\right)|\hat{\Xi}\rangle
$$

where $\lambda$ is an arbitrary real constant, and $|\hat{\Xi}\rangle$ is the dressed sliver. 
If, in addition to the above conditions, $\zeta^{\mu}$ are 'orthogonal' to the dressing vector $\xi$,

$$
\left\langle\zeta^{\mu}\left|\frac{1}{1-T^{2}}\right| \xi\right\rangle=0, \quad\left\langle\zeta^{\mu}\left|\frac{T}{1-T^{2}}\right| \xi\right\rangle=0, \quad \text { for any } \mu,
$$

it is not hard to generalize the proofs of [8], [38] and conclude that

$$
\begin{gathered}
\left|\hat{\Lambda}_{n}\right\rangle *\left|\hat{\Lambda}_{m}\right\rangle=\delta_{n, m}\left|\hat{\Lambda}_{n}\right\rangle \\
\left\langle\hat{\Lambda}_{n} \mid \hat{\Lambda}_{m}\right\rangle=\delta_{n, m}\langle\hat{\Xi} \mid \hat{\Xi}\rangle
\end{gathered}
$$

As explained in section 3, the additional conditions (7.11) are easy to comply with.

- Lump solutions. In VSFT lump solutions of any dimension have been found, [7, 8]. They are candidates to represent lower dimensional branes. By definition, they are not translational invariant in a subset of directions (the transverse ones). In order to find such solutions we cannot drop anymore the momentum dependence in the transverse directions. We therefore proceeds as follows. First we split the Lorentz indices $\mu, \nu$ into parallel ones, $\bar{\mu}, \bar{\nu}$, running from 0 to $25-k$, and transverse ones, $\alpha, \beta$ which run from $26-k$ to 25 . Next we introduce the zero modes by defining

$$
a_{0}^{(r) \alpha}=\frac{1}{2} \sqrt{b} \hat{p}^{(r) \alpha}-i \frac{1}{\sqrt{b}} \hat{x}^{(r) \alpha}, \quad a_{0}^{(r) \alpha \dagger}=\frac{1}{2} \sqrt{b} \hat{p}^{(r) \alpha}+i \frac{1}{\sqrt{b}} \hat{x}^{(r) \alpha},
$$

where $\hat{p}^{(r) \alpha}, \hat{x}^{(r) \alpha}$ are the momentum and position operator of the $r$-th string. The parameter $b$ is as in ref.[7]. The Dirac brackets for all the oscillators including the zero modes are, in the transverse directions,

$$
\left[a_{M}^{(r) \alpha}, a_{N}^{(s) \beta \dagger}\right]=\eta^{\alpha \beta} \delta^{r s} \delta_{M N}, \quad N, M \geq 0
$$

where the index $N$ denotes the couple $(0, n)$. Now we ontroduce $\left|\Omega_{b}\right\rangle$, the oscillator vacuum $\left(a_{N}^{\alpha}\left|\Omega_{b}\right\rangle=0\right.$, for $\left.N \geq 0\right)$. The relation between the momentum basis and the oscillator basis is defined by

$$
\left|\left\{p^{\alpha}\right\}\right\rangle_{123}=\left(\frac{b}{2 \pi}\right)^{\frac{k}{4}} e^{\sum_{r=1}^{3}\left(-\frac{b}{4} p_{\alpha}^{(r)} \eta^{\alpha \beta} p_{\beta}^{(r)}+\sqrt{b} a_{0}^{(r) \alpha \dagger} p_{\alpha}^{(r)}-\frac{1}{2} a_{0}^{(r) \alpha \dagger} \eta_{\alpha \beta} a_{0}^{(r) \beta \dagger}\right)}\left|\Omega_{b}\right\rangle
$$

Inserting this into (2.9) and integrating with respect to the transverse momenta one finally gets the following three strings vertex [24, []

$$
\left|V_{3}\right\rangle^{\prime}=\left|V_{3, \perp}\right\rangle^{\prime} \otimes\left|V_{3, \|}\right\rangle
$$

$\left|V_{3, \|}\right\rangle$ is the one used before this subsection (with zero momenta), while

$$
\left|V_{3, \perp}\right\rangle^{\prime}=K_{2} e^{-E^{\prime}}\left|\Omega_{b}\right\rangle
$$

where $K_{2}$ is a suitable constant and

$$
E^{\prime}=\frac{1}{2} \sum_{r, s=1}^{3} \sum_{M, N \geq 0} a_{M}^{(r) \alpha \dagger} V_{M N}^{\prime r s} a_{N}^{(s) \beta \dagger} \eta_{\alpha \beta}
$$


The vertex coefficients $V_{M N}^{\prime r s}$ to be used in the transverse directions have parallel properties to the vertex $V_{m n}^{r s}$. When multiplied by the twist matrix they give rise to matrices $X^{\prime}, X_{+}^{\prime}, X_{-}^{\prime}$ which happen to obey the same equations collected in Appendix A for the matrices $X, X_{+}, X_{-}$. Since all the calculations we have done throughout the paper depend uniquely on such properties, we can repeat everything almost verbatim. So, there will be a matrix $T^{\prime}$ given by a formula (2.18), with a normalization (2.19) and a bpz norm (2.20), where all the entries are primed. Next we introduce the dressed sliver exactly as in section 2. To this end first we define the infinite vector $\xi^{\prime}=\left\{\xi_{N}^{\prime}\right\}$ satisfying the condition

$$
\rho_{1}^{\prime} \xi^{\prime}=0, \quad \rho_{2}^{\prime} \xi^{\prime}=\xi^{\prime}
$$

and

$$
\xi^{\prime T} \frac{1}{1-T^{2}} \xi^{\prime}=1, \quad \xi^{\prime T} \frac{T^{\prime}}{1-T^{2}} \xi^{\prime}=\kappa
$$

where $\kappa$ is the same number as in (2.22). The transverse dressed sliver is defined by

$$
\left|\hat{\Xi}_{\perp}\right\rangle=\hat{\mathcal{N}}^{\prime} e^{-\frac{1}{2} a^{\dagger} \hat{S}^{\prime} a^{\dagger}}\left|\Omega_{b}\right\rangle
$$

where

$$
\hat{S}^{\prime}=S^{\prime}+R^{\prime}, \quad R_{M N}^{\prime}=\frac{1}{\kappa+1}\left(\xi_{M}^{\prime}(-1)^{N} \xi_{N}^{\prime}+\xi_{N}^{\prime}(-1)^{M} \xi_{M}^{\prime}\right)
$$

and so on. The proofs of section 3 can be repeated, given the diagonal structure of Neumann matrices with zero modes [39]. Once again we introduce a deformation parameter $\epsilon$ (the same as in section 4!) and repeat the derivations of section 5 (where D, for the transverse directions, equals $k-1$ ).

One of the most remarkable results of VSFT is the reproduction of the ratio of tensions for brane of different dimensions. It is important to verify that our regularization procedure does not alter this ratio.

It is easy to show that in the present case the ratio of tensions for brane of adjacent dimensions can be written as follows

$$
\frac{\mathcal{T}_{24-k}}{2 \pi \mathcal{T}_{25-k}}=\frac{3}{\sqrt{2 \pi b^{3}}}\left(V_{00}+\frac{b}{2}\right)^{2} \frac{\left(\operatorname{det}\left(1-X^{\prime}\right)^{3 / 4} \operatorname{det}\left(1+3 X^{\prime}\right)^{1 / 4}\right.}{\left(\operatorname{det}(1-X)^{3 / 4} \operatorname{det}(1+3 X)^{1 / 4}\right.} \cdot f(\epsilon, \kappa)
$$

The factor $f(\kappa, \epsilon)$ is due to dressing. However it is elementary to prove that this factor is actually 1. What remains is the same as in [7]. It was proven numerically [7] and analytically [31] that the ratio at the rhs of (7.23) is exactly 1, thus reproducing the expected ratio.

It goes without saying that one can easily introduce a constant background $B$ field in the transverse directions, along the lines of [36, 37].

\section{Some comments and conclusions}

There has been in the recent past some debate concerning the possibility to define finite action solutions in VSFT, 21, 23, 40]. Here we would like to clarify the relation of our 
treatment with these previous works. First of all our action does not require any overall infinite normalization, while the ones proposed by [6, 13] are singularly normalized.

Let us begin with the VSFT action proposed by Hata and Kawano 13

$$
S_{H K}[\psi]=-K\left(\frac{1}{2}\langle\psi|\mathcal{Q}| \psi\rangle+\frac{1}{3}\langle\psi \mid \psi * \psi\rangle\right)
$$

where, from both conformal and operatorial considerations, $\mathcal{Q}$ is given by [21, 13, 34]

$$
\mathcal{Q}=\frac{1}{2 i}(c(i)-c(-i))
$$

In 21] Gaiotto et al. argued that a $\mathcal{Q}$ of this kind should be obtained by a (singular) field redefinition, so that the OSFT action expanded on the tachyon vacuum would take the form

$$
S_{G R S Z}[\tilde{\psi}]=-\frac{1}{g_{0}^{2}}\left(\frac{1}{2 \epsilon}\langle\tilde{\psi}|\mathcal{Q}| \tilde{\psi}\rangle+\frac{1}{3}\langle\tilde{\psi} \mid \tilde{\psi} * \tilde{\psi}\rangle\right)
$$

where an $\epsilon \rightarrow 0$ limit is understood. Another numerical field redefinition $\tilde{\psi}=\left(g_{0}^{2} K\right)^{1 / 3} \psi$ brings $S_{G R S Z}$ to $S_{H K}$, provided we identify

$$
K=\frac{1}{g_{0}^{2} \epsilon^{3}}
$$

so that

$$
S_{H K}[\psi]=-\frac{1}{g_{0}^{2} \epsilon^{3}}\left(\frac{1}{2}\langle\psi|\mathcal{Q}| \psi\rangle+\frac{1}{3}\langle\psi \mid \psi * \psi\rangle\right)
$$

In the present work we have instead considered a finitely normalized action

$$
S[\hat{\psi}]=-\frac{1}{g_{0}^{2}}\left(\frac{1}{2}\langle\hat{\psi}|\mathcal{Q}| \hat{\psi}\rangle+\frac{1}{3}\langle\hat{\psi} \mid \hat{\psi} * \hat{\psi}\rangle\right)
$$

This action can be indeed brought to the $S_{H K}=S_{G R S Z}$ form by the operatorial field redefinition 40

$$
\psi=e^{-\frac{1}{4} \ln \epsilon\left(K_{2}-4\right)} \hat{\psi}
$$

In fact the family of operators $K_{n}=L_{n}-(-1)^{n} L_{-n}$ leaves the cubic term invariant while it acts non trivially on the kinetic term as

$$
\left[K_{2 n}, \mathcal{Q}\right]=-4 n(-1)^{n} \mathcal{Q}
$$

So, if a classical solution exists that can render our VSFT action finite (that is, such that we can find a regularization scheme in which the $b p z-$ norm can be tuned to a finite value), then something similar should be done with the singularly normalized actions (8.2, 8.4). This is in fact what Okawa found in [40] using the regularized (twisted) butterfly state [21, 10] and representing it in the non-twisted CFT. In this way he showed that one can even reabsorb the open string coupling constant into a field redefinition of the kind (8.6), so that such a coupling constant is not a free parameter.

This fact seems to mark a difference between OSFT and VSFT. In the former one cannot implement a redefinition like (8.6) keeping the ratio of the kinetic and interaction 
terms constant. Therefore in VSFT there seems to be no free parameter in the action. It is not clear to us if such a feature of VSFT is really a problem: in fact we have seen that free parameters appear effectively in the process of regularizing the classical solution, and one should note that VSFT gives the right ratios between energy densities [31, 20]. So, if a finite energy solution depending on such effective parameters is defined in such a way that they are constrained to yield the correct physical value for its tension, the tensions of all the other branes are correctly predicted.

Finally, our results in this paper may shed some light on a conclusion drawn in 40. concerning the validity of the EOM inside the action. From the formulas of Appendix $\mathrm{C}$ one can see that, if one insists in defining the dressed sliver as the strong limit of $\hat{\Psi}_{\epsilon, \tilde{\epsilon}}=\hat{\Xi}_{\epsilon} \otimes \widehat{\widetilde{\Xi}}_{\tilde{\epsilon}}$ for $\epsilon, \tilde{\epsilon} \rightarrow 1$, it follows that

$$
\lim _{\epsilon \rightarrow 1, \tilde{\epsilon} \rightarrow 1}\left\langle\hat{\Psi}_{\epsilon, \tilde{\epsilon}}|\mathcal{Q}| \hat{\Psi}_{\epsilon, \tilde{\epsilon}}\right\rangle=-\left(\frac{3}{2}\right)^{D-2} \lim _{\epsilon \rightarrow 1, \tilde{\epsilon} \rightarrow 1}\left\langle\hat{\Psi}_{\epsilon, \tilde{\epsilon}} \mid \hat{\Psi}_{\epsilon, \tilde{\epsilon}} * \hat{\Psi}_{\epsilon, \tilde{\epsilon}}\right\rangle
$$

An analogous problem is shown to exist in [40]. It was supposed there that, in order to satisfy the EOM one should take into account subleading terms in $\mathcal{Q}$, hence loosing the very appealing matter-ghost factorization of VSFT. Here we have instead attributed the mismatch (8.8) to subtleties of the limiting procedure.

\subsection{Conclusions}

In this paper we have shown that it is possible to find solutions of VSFT with finite $b p z-$ norm and action. This result was achieved by first introducing a new kind of solutions of VSFT, which we called dressed slivers. The latter is a deformation of the well-known sliver solution by the addition of a rank one projector to the Neumann matrix. The dressed sliver, introduced in this naive way, has still an ill-defined norm (and action), but one can naturally introduce a regularization parameter $\epsilon$ (which interpolates between the sliver and the dressed sliver), and tune it to the level truncation parameter $L$. This leads to a finite $b p z$-norm and action. We have seen that this regularization is far from unique. However we have also seen that most regularization prescriptions are incompatible with the EOM. Requiring the validity of the EOM we have been able to define a unique prescription. We leave to [41] the problem of testing this prescription on the open string states of the dressed sliver.

\section{Acknowledgments}

We would like to thank M.Schnabl and D.Mamone for useful discussions and suggestions. C.M. would like to thank L.F.Alday and M.Cirafici for discussions. P.P. would like to thank SISSA-ISAS (Trieste) and Dipartimento di Fisica Teorica (Università degli Studi di Torino) for their kind hospitality and stimulating atmosphere during this research. This research was supported by the Italian MIUR under the program "Teoria dei Campi, Superstringhe e Gravità". 


\section{Appendix}

\section{A. A collection of well-known formulae}

In this Appendix we collect some useful results and formulas involving the matrices of the three strings vertex coefficients.

To start with, we recall that

- (i) $V_{n m}^{r s}$ are symmetric under simultaneous exchange of the two couples of indices;

- (ii) they are endowed with the property of cyclicity in the $r, s$ indices, i.e. $V^{r s}=$ $V^{r+1, s+1}$, where $r, s=4$ is identified with $r, s=1$.

Next, using the twist matrix $C\left(C_{m n}=(-1)^{m} \delta_{m n}\right)$, we define

$$
X^{r s} \equiv C V^{r s}, \quad r, s=1,2,
$$

These matrices are often rewritten in the following way $X^{11}=X, X^{12}=X_{+}, X^{21}=X_{-}$. They commute with one another

$$
\left[X^{r s}, X^{r^{\prime} s^{\prime}}\right]=0
$$

moreover

$$
C V^{r s}=V^{s r} C, \quad C X^{r s}=X^{s r} C
$$

Next we quote some useful identities:

$$
\begin{aligned}
& X+X_{+}+X_{-}=1 \\
& X_{+} X_{-}=(X)^{2}-X \\
& \left(X_{+}\right)^{2}+\left(X_{-}\right)^{2}=1-(X)^{2} \\
& \left(X_{+}\right)^{3}+\left(X_{-}\right)^{3}=2(X)^{3}-3(X)^{2}+1
\end{aligned}
$$

and

$$
\frac{1-T X}{1-X}=\frac{1}{1-T}, \quad \frac{X}{1-X}=\frac{T}{(1-T)^{2}}
$$

Using these one can show, for instance, that

$$
\begin{aligned}
& \mathcal{K}^{-1}=\frac{1}{(1+T)(1-X)}\left(\begin{array}{cc}
1-T X & T X_{+} \\
T X_{-} & 1-T X
\end{array}\right) \\
& \mathcal{M K}^{-1}=\frac{1}{(1+T)(1-X)}\left(\begin{array}{cc}
(1-T X) X & X_{+} \\
X_{-} & (1-T X) X
\end{array}\right)
\end{aligned}
$$

Another ingredient we need is given by the Fock space projectors

$$
\begin{aligned}
& \rho_{1}=\frac{1}{(1+T)(1-X)}\left[X_{+}(1-T X)+T\left(X_{-}\right)^{2}\right] \\
& \rho_{2}=\frac{1}{(1+T)(1-X)}\left[X_{-}(1-T X)+T\left(X_{+}\right)^{2}\right]
\end{aligned}
$$


They satisfy

$$
\rho_{1}^{2}=\rho_{1}, \quad \rho_{2}^{2}=\rho_{2}, \quad \rho_{1}+\rho_{2}=1, \quad \rho_{1} \rho_{2}=0
$$

i.e. they project onto orthogonal subspaces. Moreover,

$$
\rho_{1}^{T}=\rho_{1}=C \rho_{2} C, \quad \rho_{2}^{T}=\rho_{2}=C \rho_{1} C .
$$

where $^{T}$ represents matrix transposition. As was shown in [8, 12], $\rho_{1}, \rho_{2}$ projects out half the string modes. Using these projectors one can prove that

$$
\left(X_{+}, X_{-}\right) \mathcal{K}^{-1}=\left(\rho_{1}, \rho_{2}\right), \quad \mathcal{M} \mathcal{K}^{-1} \mathcal{T}\left(\begin{array}{l}
X_{-} \\
X_{+}
\end{array}\right)=\left(\begin{array}{l}
T X \rho_{2}+T X_{+} \rho_{1} \\
T X_{-} \rho_{2}+T X \rho_{1}
\end{array}\right)
$$

which are used throughout the paper.

The following relations are often useful

$$
\rho_{1} X_{+}+\rho_{2} X_{-}=1-X T, \quad \rho_{1} X_{-}+\rho_{2} X_{+}=X(T-1)
$$

In the ghost sector matrices $\tilde{X}, \tilde{X}_{ \pm}$satisfy all relations above. In particular one can define the half-string projectors $\tilde{\rho}_{1}, \tilde{\rho}_{2}$ as in (A.7, A.8) and verify that they satisfy the same relations as the matter projectors. For the manipulations with zero-modes it is useful here to define the vectors

$$
\left(\widetilde{\mathbf{v}}_{0}\right)_{n}=\widetilde{V}_{n 0}^{r, r}, \quad\left(\widetilde{\mathbf{v}}_{ \pm}\right)_{n}=\widetilde{V}_{n 0}^{r, r \pm 1}
$$

which satisfy

$$
\widetilde{\mathbf{v}}_{0}=(1-\widetilde{X}) \mathbf{f}, \quad \widetilde{\mathbf{v}}_{ \pm}=-\widetilde{X}_{\mp} \mathbf{f}
$$

where $\mathbf{f}=\left\{f_{n}\right\}$ is given by

$$
f_{n}=\cos \left(\frac{n \pi}{2}\right) .
$$

This vector $\mathbf{f}$ appears in the expression for the kinetic operator $\mathcal{Q}$ :

$$
\mathcal{Q}=c_{0}+\sum_{n=1}^{\infty} f_{n}\left(c_{n}+(-1)^{n} c_{n}^{\dagger}\right) .
$$

\section{B. Evaluation of determinants}

This section is devoted to the evaluation of determinants which appear in calculations involving dressed slivers. Here we deal only with the matter determinants, but the same results hold for the corresponding ghost determinants.

B.1 $\operatorname{Det}\left(1-\hat{\mathcal{T}}_{\epsilon} \mathcal{M}\right)$

First of all we consider

$$
\left(1-\hat{\mathcal{T}}_{\epsilon} \mathcal{M}\right)^{-1} \mathcal{P}=\mathcal{K}^{-1}\left(1-\epsilon \mathcal{P} \mathcal{M K}^{-1}\right)^{-1} \mathcal{P}
$$

This matrix can be exactly computed from

$$
\mathcal{P} \mathcal{M K}^{-1} \mathcal{P}=\left(\begin{array}{cc}
\kappa & \rho_{1}-\kappa \rho_{2} \\
\rho_{2}-\kappa \rho_{1} & \kappa
\end{array}\right) \mathcal{P}
$$


We have in fact

$$
\left(1-\epsilon \mathcal{P} \mathcal{M} \mathcal{K}^{-1}\right)^{-1} \mathcal{P}=\sum_{n=0}^{\infty}\left(\epsilon \mathcal{P} \mathcal{M} \mathcal{K}^{-1}\right)^{n} \mathcal{P}
$$

Using the properties of the $\rho$ projectors, defined in the previous appendix, we can easily show that

$$
\left(\epsilon \mathcal{P} \mathcal{M K}^{-1}\right)^{n} \mathcal{P}=\frac{\epsilon^{n}}{(\kappa+1)^{n}}\left(\begin{array}{cc}
A(n) & B(n)\left(\rho_{1}-\kappa \rho_{2}\right) \\
B(n)\left(\rho_{2}-\kappa \rho_{1}\right) & A(n)
\end{array}\right) \mathcal{P}
$$

where

$$
\begin{aligned}
A(n) & =\sum_{l=0}^{\left[\frac{n}{2}\right]}(-1)^{l} k^{n-l}\left(\begin{array}{c}
n \\
2 l
\end{array}\right) \\
B(n) & =\sum_{p=0}^{\left[\frac{n-1}{2}\right]}(-1)^{p} k^{n-p-1}\left(\begin{array}{c}
n \\
2 p+1
\end{array}\right)
\end{aligned}
$$

Now we exchange the order of summations

$$
\begin{gathered}
\sum_{n=0}^{\infty} \sum_{l=0}^{\left[\frac{n}{2}\right]}=\sum_{l=0}^{\infty} \sum_{n=2 l}^{\infty} \\
\sum_{n=0}^{\infty} \sum_{p=0}^{\left[\frac{n-1}{2}\right]}=\sum_{p=0}^{\infty} \sum_{n=2 p+1}^{\infty}
\end{gathered}
$$

and use the resummation formula

$$
\sum_{n=l}^{\infty}\left(\begin{array}{l}
n \\
l
\end{array}\right) \frac{p^{n-l}}{q^{n}}=\frac{q}{(q-p)^{l+1}}
$$

With standard algebraic manipulations, we get

$$
\left(1-\epsilon \mathcal{P} \mathcal{M K}^{-1}\right)^{-1} \mathcal{P}=\frac{\kappa+1}{(\kappa-\epsilon \kappa+1)^{2}+\epsilon^{2} \kappa}\left(\begin{array}{cc}
\kappa-\epsilon \kappa+1 & \epsilon\left(\rho_{1}-\kappa \rho_{2}\right) \\
\epsilon\left(\rho_{2}-\kappa \rho_{1}\right) & \kappa-\epsilon \kappa+1
\end{array}\right) \mathcal{P}
$$

In order to compute $\operatorname{Tr} \ln \left(1-\epsilon \mathcal{P} \mathcal{M} \mathcal{K}^{-1}\right)$ we first consider

$$
\begin{aligned}
& \frac{d}{d \epsilon} \operatorname{Tr} \ln \left(1-\epsilon \mathcal{P} \mathcal{M} \mathcal{K}^{-1}\right)=-\operatorname{Tr}\left[\left(1-\epsilon \mathcal{P} \mathcal{M K}^{-1}\right)^{-1} \mathcal{P} \mathcal{M} \mathcal{K}^{-1}\right] \\
& =-\operatorname{Tr}\left[\frac{\kappa+1}{(\kappa-\epsilon \kappa+1)^{2}+\epsilon^{2} \kappa}\left(\begin{array}{cc}
\kappa-\epsilon \kappa+1 & \epsilon\left(\rho_{1}-\kappa \rho_{2}\right) \\
\epsilon\left(\rho_{2}-\kappa \rho_{1}\right) & \kappa-\epsilon \kappa+1
\end{array}\right) \mathcal{P} \mathcal{M} \mathcal{K}^{-1}\right] \\
& =-\frac{\kappa+1}{(\kappa-\epsilon \kappa+1)^{2}+\epsilon^{2} \kappa} \operatorname{tr}\left[2(\kappa-\epsilon \kappa+1) P \frac{T}{1-T^{2}}-\epsilon P \frac{T}{1-T^{2}}-\epsilon \kappa P \frac{1}{1-T^{2}}\right] \\
& =-\frac{2(\kappa+1)}{(\kappa-\epsilon \kappa+1)^{2}+\epsilon^{2} \kappa}\left(2(\kappa-\epsilon \kappa+1) \frac{\kappa}{\kappa+1}-\epsilon \frac{\kappa}{\kappa+1}-\epsilon \kappa \frac{1}{\kappa+1}\right) \\
& =-4 \frac{\kappa(\kappa+1)(1-\epsilon)}{(\kappa-\epsilon \kappa+1)^{2}+\epsilon^{2} \kappa}
\end{aligned}
$$


Hence we get

$$
\operatorname{Tr} \ln \left(1-\epsilon \mathcal{P} \mathcal{M K}^{-1}\right)=-4 \int_{0}^{\epsilon} d \epsilon^{\prime} \frac{\kappa(\kappa+1)\left(1-\epsilon^{\prime}\right)}{\left(\kappa-\epsilon^{\prime} \kappa+1\right)^{2}+\epsilon^{\prime 2} \kappa}=2 \ln \frac{1+(1-\epsilon)^{2} \kappa}{\kappa+1}
$$

Collecting all the contributions we finally obtain

$$
\operatorname{Det}\left(1-\hat{\mathcal{T}}_{\epsilon} \mathcal{M}\right)=\left(\frac{1+(1-\epsilon)^{2} \kappa}{\kappa+1}\right)^{2} \operatorname{Det}(1-\mathcal{T} \mathcal{M})
$$

B.2 $\operatorname{Det}\left(1-\hat{\mathcal{T}}_{\epsilon_{1} \epsilon_{2}} \mathcal{M}\right)$

To compute this determinant we use the same strategy as before, that is we first compute

$$
\left(1-\mathcal{P}_{\epsilon_{1} \epsilon_{2}} \mathcal{M} \mathcal{K}^{-1}\right)^{-1} \mathcal{P}_{\epsilon_{1} \epsilon_{2}}=\left(\begin{array}{cc}
A & B\left(\rho_{1}-\kappa \rho_{2}\right) \\
D\left(\rho_{2}-\kappa \rho_{1}\right) & D
\end{array}\right) \mathcal{P}_{\epsilon_{1} \epsilon_{2}}
$$

where $A, B, C, D$ are to be determined. Moreover we have defined

$$
\begin{aligned}
& \mathcal{P}_{\epsilon_{1} \epsilon_{2}}=\left(\begin{array}{cc}
\epsilon_{1} & 0 \\
0 & \epsilon_{2}
\end{array}\right) P \\
& \hat{\mathcal{T}}_{\epsilon_{1} \epsilon_{2}}=\left(\begin{array}{cc}
\hat{T}_{\epsilon_{1}} & 0 \\
0 & \hat{T}_{\epsilon_{2}}
\end{array}\right)
\end{aligned}
$$

The constant $A, B, C, D$ can be easily determined by imposing $\left(1-\mathcal{P}_{\epsilon_{1} \epsilon_{2}} \mathcal{M} \mathcal{K}^{-1}\right)^{-1} \mathcal{P}_{\epsilon_{1} \epsilon_{2}}$ to give back $\mathcal{P}_{\epsilon_{1} \epsilon_{2}}$ when multiplied on the left by $\left(1-\mathcal{P}_{\epsilon_{1} \epsilon_{2}} \mathcal{M} \mathcal{K}^{-1}\right)$. The procedure is straightforward and gives the result

$$
\left(1-\mathcal{P}_{\epsilon_{1} \epsilon_{2}} \mathcal{M K}^{-1}\right)^{-1} \mathcal{P}_{\epsilon_{1} \epsilon_{2}}=\frac{1}{1+\left(1-\epsilon_{1}\right)\left(1-\epsilon_{2}\right) \kappa}\left(\begin{array}{cc}
\kappa+1-\epsilon_{2} \kappa & \epsilon_{1}\left(\rho_{1}-\kappa \rho_{2}\right) \\
\epsilon_{2}\left(\rho_{2}-\kappa \rho_{1}\right) & \kappa+1-\epsilon_{1} \kappa
\end{array}\right) \mathcal{P}_{\epsilon_{1} \epsilon_{2}}
$$

Now we come to the computation of the determinant

$$
\operatorname{Det}\left(1-\hat{\mathcal{T}}_{\epsilon_{1} \epsilon_{2}} \mathcal{M}\right)=\operatorname{Det}(1-\mathcal{T} \mathcal{M}) \exp \left(\operatorname{Tr} \ln \left(1-\mathcal{P}_{\epsilon_{1} \epsilon_{2}} \mathcal{M} \mathcal{K}^{-1}\right)\right)
$$

To compute the exponent of the second factor in the rhs we use the same strategy as before, namely we consider

$$
\begin{aligned}
& \frac{d}{d x} \operatorname{Tr} \ln \left(1-x \mathcal{P}_{\epsilon_{1} \epsilon_{2}} \mathcal{M} \mathcal{K}^{-1}\right)=-\operatorname{Tr}\left[\left(1-x \mathcal{P}_{\epsilon_{1} \epsilon_{2}} \mathcal{M} \mathcal{K}^{-1}\right)^{-1} \mathcal{P}_{\epsilon_{1} \epsilon_{2}} \mathcal{M} \mathcal{K}^{-1}\right] \\
& =-\operatorname{Tr}\left[\frac{1}{x}\left(1-\mathcal{P}_{x \epsilon_{1}, x \epsilon_{2}} \mathcal{M} \mathcal{K}^{-1}\right)^{-1} \mathcal{P}_{x \epsilon_{1}, x \epsilon_{2}} \mathcal{M} \mathcal{K}^{-1}\right] \\
& =-\operatorname{Tr}\left[\frac{1}{x} \frac{1}{1+\left(1-\epsilon_{1}\right)\left(1-\epsilon_{2}\right) \kappa}\left(\begin{array}{cc}
\kappa+1-x \epsilon_{2} \kappa & x \epsilon_{1}\left(\rho_{1}-\kappa \rho_{2}\right) \\
x \epsilon_{2}\left(\rho_{2}-\kappa \rho_{1}\right) & \kappa+1-x \epsilon_{1} \kappa
\end{array}\right) \mathcal{P}_{x \epsilon_{1}, x \epsilon_{2}} \mathcal{M} \mathcal{K}^{-1}\right] \\
& =-2 \frac{\left(\epsilon_{1}+\epsilon_{2}\right) \kappa-2 x \epsilon_{1} \epsilon_{2} \kappa}{1+\left(1-x \epsilon_{1}\right)\left(1-x \epsilon_{2}\right) \kappa}
\end{aligned}
$$


where the same manipulations as in (B.9) have been used. Then we perform the simple integration

$$
\operatorname{Tr} \ln \left(1-\mathcal{P}_{\epsilon_{1} \epsilon_{2}} \mathcal{M K}^{-1}\right)=-2 \int_{0}^{1} d x \frac{\left(\epsilon_{1}+\epsilon_{2}\right) \kappa-2 x \epsilon_{1} \epsilon_{2} \kappa}{1+\left(1-x \epsilon_{1}\right)\left(1-x \epsilon_{2}\right) \kappa}=2 \ln \left(\frac{1+\left(1-\epsilon_{1}\right)\left(1-\epsilon_{2}\right) \kappa}{\kappa+1}\right)
$$

Therefore we have obtained

$$
\operatorname{Det}\left(1-\hat{\mathcal{T}}_{\epsilon_{1} \epsilon_{2}} \mathcal{M}\right)=\left(\frac{1+\left(1-\epsilon_{1}\right)\left(1-\epsilon_{2}\right) \kappa}{\kappa+1}\right)^{2} \operatorname{Det}(1-\mathcal{T} \mathcal{M})
$$

B.3 $\operatorname{det}\left(1-\hat{T}_{\epsilon}^{2}\right)$

We have

$$
\operatorname{det}\left(1-\hat{T}_{\epsilon}^{2}\right)=\operatorname{det}\left(1-\hat{T}_{\epsilon}\right) \operatorname{det}\left(1+\hat{T}_{\epsilon}\right)
$$

We compute the two factors separately

$$
\operatorname{det}\left(1-\hat{T}_{\epsilon}\right)=\operatorname{det}\left(1-\frac{\epsilon}{1-T} P\right) \operatorname{det}(1-T)
$$

For the first factor in the rhs we have

$$
\begin{aligned}
\operatorname{det}\left(1-\frac{\epsilon}{1-T} P\right)= & \exp \left(\operatorname{tr} \ln \left(1-\frac{\epsilon}{1-T} P\right)\right) \\
& =\exp \left(-\sum_{n=1}^{\infty} \frac{1}{n} \operatorname{tr}\left(\frac{\epsilon}{1-T} P\right)^{n}\right)=\exp \left(-2 \sum_{n=1}^{\infty} \frac{\epsilon^{n}}{n(\kappa+1)^{n}}\left\langle\xi\left|\frac{1}{1-T}\right| \xi\right\rangle^{n}\right) \\
& =\exp \left(-2 \sum_{n=1}^{\infty} \frac{\epsilon^{n}}{n(\kappa+1)^{n}}(\kappa+1)^{n}\right)=\exp (2 \ln (1-\epsilon)) \\
& =(1-\epsilon)^{2}
\end{aligned}
$$

So we have

$$
\operatorname{det}\left(1-\hat{T}_{\epsilon}\right)=(1-\epsilon)^{2} \operatorname{det}(1-T)
$$

Now let's turn to the second factor in (B.21)

$$
\operatorname{det}\left(1+\hat{T}_{\epsilon}\right)=\operatorname{det}\left(1+\frac{\epsilon}{1+T} P\right) \operatorname{det}(1+T)
$$

Computing as in $(\overline{B .23})$ we obtain

$$
\operatorname{det}\left(1+\frac{\epsilon}{1+T} P\right)=\left(\frac{\kappa+1-\epsilon(\kappa-1)}{\kappa+1}\right)^{2}
$$

giving the result

$$
\operatorname{det}\left(1+\hat{T}_{\epsilon}\right)=\left(\frac{\kappa+1-\epsilon(\kappa-1)}{\kappa+1}\right)^{2} \operatorname{det}(1+T)
$$

Collecting the two results $(\mathrm{B} .23, \mathrm{~B} .26)$ we get

$$
\operatorname{det}\left(1-\hat{T}_{\epsilon}^{2}\right)=(1-\epsilon)^{2}\left(\frac{\kappa+1-\epsilon(\kappa-1)}{\kappa+1}\right)^{2} \operatorname{det}\left(1-T^{2}\right)
$$


B.4 $\operatorname{det}\left(1-\hat{T}_{\epsilon_{1}} \hat{T}_{\epsilon_{2}}\right)$

First of all we decompose

$$
\begin{aligned}
& 1-\hat{T}_{\epsilon_{1}} \hat{T}_{\epsilon_{2}}=\left(1-\hat{T}_{\epsilon_{1}}\right)\left(1+\hat{T}_{\epsilon_{2}}\right)+\hat{T}_{\epsilon_{1}}-\hat{T}_{\epsilon_{2}} \\
& =\left(1-\hat{T}_{\epsilon_{1}}\right)\left(1+\left(\epsilon_{1}-\epsilon_{2}\right)\left(1-\hat{T}_{\epsilon_{1}}\right)^{-1} P\left(1+\hat{T}_{\epsilon_{2}}\right)^{-1}\right)\left(1+\hat{T}_{\epsilon_{2}}\right)
\end{aligned}
$$

So we have

$$
\operatorname{det}\left(1-\hat{T}_{\epsilon_{1}} \hat{T}_{\epsilon_{2}}\right)=\operatorname{det}\left(1-\hat{T}_{\epsilon_{1}}\right) \operatorname{det}\left(1+\hat{T}_{\epsilon_{2}}\right) \operatorname{det}\left(1+\left(\epsilon_{1}-\epsilon_{2}\right)\left(1-\hat{T}_{\epsilon_{1}}\right)^{-1} P\left(1+\hat{T}_{\epsilon_{2}}\right)^{-1}\right)
$$

We need to compute the third factor in rhs

$$
\begin{aligned}
& \operatorname{det}\left(1+\left(\epsilon_{1}-\epsilon_{2}\right)\left(1-\hat{T}_{\epsilon_{1}}\right)^{-1} P\left(1+\hat{T}_{\epsilon_{2}}\right)^{-1}\right)= \\
& =\exp \left(\operatorname{tr} \ln \left(1+\left(\epsilon_{1}-\epsilon_{2}\right)\left(1-\hat{T}_{\epsilon_{1}}\right)^{-1} P\left(1+\hat{T}_{\epsilon_{2}}\right)^{-1}\right)\right) \\
& =\exp \left(-2 \sum_{n=1}^{\infty} \frac{(-1)^{n}}{n}\left(\frac{\epsilon_{1}-\epsilon_{2}}{\kappa+1}\right)^{n}\left\langle\xi\left|\left(1+\hat{T}_{\epsilon_{2}}\right)^{-1}\left(1-\hat{T}_{\epsilon_{1}}\right)^{-1}\right| \xi\right\rangle^{n}\right)
\end{aligned}
$$

where the factor 2 is to take into account the (equal) contributions of $\xi$ and $C \xi$ which constitute $P$, so from now on only the contribution of $\xi$ is needed to be considered. Then we decompose $\left(1+\hat{T}_{\epsilon_{2}}\right)^{-1}$ and $\left(1-\hat{T}_{\epsilon_{1}}\right)^{-1}$ as

$$
\begin{aligned}
& \left(1+\hat{T}_{\epsilon_{2}}\right)^{-1}=\left(\left(1+\epsilon_{2} P \frac{1}{1+T}\right)(1+T)\right)^{-1}=\frac{1}{1+T} \sum_{m=0}^{\infty}\left(\frac{-\epsilon_{2}}{\kappa+1}\right)^{m}\left(|\xi\rangle\langle\xi| \frac{1}{1+T}\right)^{m} \\
& \left(1-\hat{T}_{\epsilon_{1}}\right)^{-1}=\left((1-T)\left(1+\frac{1}{1-T} \epsilon_{1} P\right)\right)^{-1}=\sum_{p=0}^{\infty}\left(\frac{\epsilon_{1}}{\kappa+1}\right)^{p}\left(\frac{1}{1-T}(|\xi\rangle\langle\xi|)^{p} \frac{1}{1-T}\right.
\end{aligned}
$$

So we get

$$
\begin{aligned}
& \left\langle\xi\left|\left(1+\hat{T}_{\epsilon_{2}}\right)^{-1}\left(1-\hat{T}_{\epsilon_{1}}\right)^{-1}\right| \xi\right\rangle^{n} \\
& =\left(\sum_{m=0}^{\infty}\left(\frac{-\epsilon_{2}}{\kappa+1}\right)^{m}\left\langle\xi\left|\frac{1}{1+T}\right| \xi\right\rangle^{m}\left\langle\xi\left|\frac{1}{1-T^{2}}\right| \xi\right\rangle \sum_{p=0}^{\infty}\left(\frac{\epsilon_{1}}{\kappa+1}\right)^{p}\left\langle\xi\left|\frac{1}{1-T}\right| \xi\right\rangle^{p}\right)^{n} \\
& =\left(\frac{\kappa+1}{\left(\kappa+1-\epsilon_{2}(\kappa-1)\right)\left(1-\epsilon_{1}\right)}\right)^{n}
\end{aligned}
$$

plugging this in $(\overline{\mathrm{B} .31})$, we get

$$
\begin{aligned}
& \operatorname{det}\left(1+\left(\epsilon_{1}-\epsilon_{2}\right)\left(1-\hat{T}_{\epsilon_{1}}\right)^{-1} P\left(1+\hat{T}_{\epsilon_{2}}\right)^{-1}\right) \\
& =\exp \left(-2 \sum_{n=1}^{\infty} \frac{1}{n}\left(\frac{\epsilon_{2}-\epsilon_{1}}{\left(1-\epsilon_{1}\right)\left(\kappa+1-\epsilon_{2}(\kappa-1)\right.}\right)^{n}\right) \\
& =\left(1-\frac{\epsilon_{2}-\epsilon_{1}}{\left(1-\epsilon_{1}\right)\left(\kappa+1-\epsilon_{2}(\kappa-1)\right.}\right)^{2}
\end{aligned}
$$

From (B.30), using (B.24, B.27), we finally get 


$$
\begin{aligned}
& \operatorname{det}\left(1-\hat{T}_{\epsilon_{1}} \hat{T}_{\epsilon_{2}}\right)=\operatorname{det}\left(1-T^{2}\right)\left(1-\left(\epsilon_{1}+\epsilon_{2}\right) \frac{\kappa}{\kappa+1}+\epsilon_{1} \epsilon_{2} \frac{\kappa-1}{\kappa+1}\right)^{2} \\
& =\operatorname{det}\left(1-T^{2}\right)\left[\frac{\epsilon_{1} \epsilon_{2}}{\kappa+1}\left(1-\frac{1}{\epsilon_{1} \star \epsilon_{2}}\right)\right]^{2} \\
& =\operatorname{det}\left(1-\hat{T}_{\epsilon_{1}}^{2}\right)^{\frac{1}{2}} \operatorname{det}\left(1-\hat{T}_{\epsilon_{2}}^{2}\right)^{\frac{1}{2}}\left(1-\frac{\epsilon_{2}-\epsilon_{1}}{\left(1-\epsilon_{1}\right)\left(\kappa+1-\epsilon_{2}(\kappa-1)\right.}\right)\left(1-\frac{\epsilon_{1}-\epsilon_{2}}{\left(1-\epsilon_{2}\right)\left(\kappa+1-\epsilon_{1}(\kappa-1)\right.}\right)
\end{aligned}
$$

Note that the last two factors in $r h s$ of the last line approach 1 as $\epsilon_{1} \rightarrow \epsilon_{2}$.

\section{Limit prescriptions}

\section{C.1 Double limit}

In this appendix we analyse various limits of the quantity

$$
\left\langle\hat{\Xi}_{\epsilon_{1}} \mid \hat{\Xi}_{\epsilon_{2}}\right\rangle=\frac{\hat{\mathcal{N}}_{\epsilon_{1}} \hat{\mathcal{N}}_{\epsilon_{2}}}{\operatorname{det}\left(1-\hat{S}_{\epsilon_{1}} \hat{S}_{\epsilon_{2}}\right)}\langle 0 \mid 0\rangle
$$

when $\epsilon_{1}, \epsilon_{2} \rightarrow 1$. We recall that $0 \leq \epsilon_{1}, \epsilon_{2} \leq 1$.

Since $\operatorname{det}\left(1-\hat{S}_{\epsilon_{1}} \hat{S}_{\epsilon_{2}}\right)=\operatorname{det}\left(1-\hat{S}_{\epsilon_{2}} \hat{S}_{\epsilon_{1}}\right)$ and

$$
\operatorname{det}\left(1-\hat{S}_{\epsilon_{1}} \hat{S}_{\epsilon_{2}}\right)=\left(\operatorname{det}\left(1-\hat{T}_{\epsilon_{1}}\right) \operatorname{det}\left(1+\hat{T}_{\epsilon_{2}}\right) \operatorname{det}\left(1+\left(\epsilon_{1}-\epsilon_{2}\right) P \frac{1}{\left(1-\hat{T}_{\epsilon_{1}}\right)\left(1+\hat{T}_{\epsilon_{2}}\right)}\right)\right)^{\frac{1}{2}}
$$

it is convenient to symmetrize the result. One gets

$$
\begin{aligned}
& \operatorname{det}\left(1-\hat{S}_{\epsilon_{1}} \hat{S}_{\epsilon_{2}}\right)=\left(\operatorname{det}\left(1-\hat{T}_{\epsilon_{1}}^{2}\right) \operatorname{det}\left(1-\hat{T}_{\epsilon_{2}}^{2}\right)\right)^{\frac{1}{2}} \\
& \cdot\left(\operatorname{det}\left(1+\left(\epsilon_{1}-\epsilon_{2}\right) P \frac{1}{\left(1-\hat{T}_{\epsilon_{1}}\right)\left(1+\hat{T}_{\epsilon_{2}}\right)}\right) \operatorname{det}\left(1+\left(\epsilon_{2}-\epsilon_{1}\right) P \frac{1}{\left(1-\hat{T}_{\epsilon_{2}}\right)\left(1+\hat{T}_{\epsilon_{1}}\right)}\right)\right)^{\frac{1}{2}}
\end{aligned}
$$

Using the results of Appendix B.4 this can be rewritten as

$$
\begin{aligned}
& \operatorname{det}\left(1-\hat{S}_{\epsilon_{1}} \hat{S}_{\epsilon_{2}}\right)=\operatorname{det}\left(1-\hat{T}_{\epsilon_{1}}^{2}\right)^{\frac{1}{2}} \operatorname{det}\left(1-\hat{T}_{\epsilon_{2}}^{2}\right)^{\frac{1}{2}} \\
& \cdot\left(1-\frac{\epsilon_{2}-\epsilon_{1}}{\left(1-\epsilon_{1}\right)\left(\kappa+1-\epsilon_{2}(\kappa-1)\right.}\right)\left(1-\frac{\epsilon_{1}-\epsilon_{2}}{\left(1-\epsilon_{2}\right)\left(\kappa+1-\epsilon_{1}(\kappa-1)\right.}\right)
\end{aligned}
$$

Therefore, collecting the previous results,

$$
\begin{gathered}
\frac{1}{\langle 0 \mid 0\rangle}\left\langle\hat{\Xi}_{\epsilon_{1}} \mid \hat{\Xi}_{\epsilon_{2}}\right\rangle=\left(\frac{\hat{\mathcal{N}}_{\epsilon_{1}}}{\sqrt{\operatorname{det}\left(1-\hat{S}_{\epsilon_{1}}^{2}\right)}}\right)^{\frac{D}{2}}\left(\frac{\hat{\mathcal{N}}_{\epsilon_{2}}}{\sqrt{\operatorname{det}\left(1-\hat{S}_{\epsilon_{2}}^{2}\right)}}\right)^{\frac{D}{2}} \\
\cdot\left(\frac{\left(1-\epsilon_{1}\right)\left(1-\epsilon_{2}\right)\left(\kappa+1-\epsilon_{1}(\kappa-1)\right)\left(\kappa+1-\epsilon_{2}(k-1)\right)}{\left(\kappa+1-\left(\epsilon_{1}+\epsilon_{2}\right) \kappa+\epsilon_{1} \epsilon_{2}(\kappa-1)\right)^{2}}\right)^{\frac{D}{2}}
\end{gathered}
$$


When $\epsilon_{1}$ and $\epsilon_{2}$ are in the vicinity of 1 , this simplifies as follows

$$
\left(\frac{\operatorname{det}(1-\Sigma \mathcal{V})}{\sqrt{\operatorname{det}\left(1-S^{2}\right)}}\right)^{D}\left(\frac{1}{4(\kappa+1)^{2}}\right)^{\frac{D}{2}}\left(\frac{4}{\left(\kappa\left(1-\epsilon_{1}\right)\left(1-\epsilon_{2}\right)+1-\epsilon_{1} \epsilon_{2}\right)^{2}}\right)^{\frac{D}{2}}+\ldots
$$

where dots denote non-leading terms. It is useful to change parametrization of $\epsilon_{1}, \epsilon_{2}$ as follows

$$
1-\epsilon_{1}=r \cos \theta \quad 1-\epsilon_{2}=r \sin \theta, \quad 0 \leq \theta \leq \pi / 2
$$

Then (C.5) becomes

$$
\left(\frac{\operatorname{det}(1-\Sigma \mathcal{V})}{\sqrt{\operatorname{det}\left(1-S^{2}\right)}}\right)^{D}\left(\frac{1}{(\kappa+1)^{2}}\right)^{\frac{D}{2}}\left(\frac{1}{r^{2}} \frac{1}{(\sin \theta+\cos \theta)^{2}}\right)^{\frac{D}{2}}+\ldots
$$

The function $(\sin \theta+\cos \theta)^{-2}$ varies between 1 and $1 / 2$, with a minimum at $\theta=\pi / 4$, which corresponds to $\epsilon_{1}=\epsilon_{2}=\epsilon(r=\sqrt{2}(1-\epsilon))$, and maxima at $\theta=0, \pi / 2$, which correspond to $\epsilon_{1}=r, \epsilon_{2}=0$ and $\epsilon_{1}=0, \epsilon_{2}=r$.

These are the two possibilities considered in section 5 . The first corresponds to $\epsilon_{1}=$ $\epsilon_{2}=\epsilon$, the second corresponds to the ordered limit. In between there are of course infinite many possibilities, giving rise to different rescalings of the number $s$.

\section{C.2 Triple limit}

We discuss here the rhs of eq.(5.12). We start with calculating an explicit formula for $\left\langle\hat{\Xi}_{\epsilon_{1}} \mid \hat{\Xi}_{\epsilon_{2}} * \hat{\Xi}_{\epsilon_{3}}\right\rangle$,

$$
\begin{aligned}
& \left\langle\hat{\Xi}_{\epsilon_{1}} \mid \hat{\Xi}_{\epsilon_{2}} * \hat{\Xi}_{\epsilon_{3}}\right\rangle=\left\langle\hat{\Xi}_{\epsilon_{1}} \mid \hat{\Xi}_{\epsilon_{2} \star \epsilon_{3}}\right\rangle=\left(\frac{\hat{\mathcal{N}}_{\epsilon_{1}} \hat{\mathcal{N}}_{\epsilon_{2} \star \epsilon_{3}}}{\sqrt{1-\hat{T}_{\epsilon_{1}} \hat{T}_{\epsilon_{2} \star \epsilon_{3}}}}\right)^{D} \\
& \sim\left(\frac{\operatorname{Det}(1-\mathcal{T} \mathcal{M})}{\sqrt{1-T^{2}}}\right)^{D} \frac{1}{(1+\kappa)^{D}}\left(1+\kappa-\left(\epsilon_{1}+\epsilon_{2} \star \epsilon_{3}\right) \kappa+\epsilon_{1} \epsilon_{2} \star \epsilon_{3}(\kappa-1)\right)^{-D} \\
& \sim\left(\frac{\operatorname{Det}(1-\mathcal{T} \mathcal{M})}{\sqrt{1-T^{2}}}\right)^{D} \frac{1}{(1+\kappa)^{D}} \\
& \cdot\left(\kappa^{2}\left(1-\epsilon_{1}\right)\left(1-\epsilon_{2}\right)\left(1-\epsilon_{3}\right)+\kappa\left(2-\epsilon_{1}-\epsilon_{2}-\epsilon_{3}+\epsilon_{1} \epsilon_{2} \epsilon_{3}\right)+1-\epsilon_{1} \epsilon_{2} \epsilon_{3}\right)^{-D}
\end{aligned}
$$

where we have kept only the dominant term for $\epsilon_{1}, \epsilon_{2}, \epsilon_{3}$ near 1 . Now let us introduce the parametrization

$$
1-\epsilon_{1}=r \cos \theta, \quad 1-\epsilon_{2}=r \sin \theta \cos \varphi, \quad 1-\epsilon_{2}=r \sin \theta \sin \varphi
$$

where $0 \leq \theta \leq \pi / 2,0 \leq \varphi \leq \pi / 2$. Then (C.8) becomes (keeping only the dominant term)

$$
\begin{aligned}
& \left\langle\hat{\Xi}_{\epsilon_{1}} \mid \hat{\Xi}_{\epsilon_{2}} * \hat{\Xi}_{\epsilon_{3}}\right\rangle \\
& \sim\left(\frac{\operatorname{Det}(1-\mathcal{T} \mathcal{M})}{\sqrt{1-T^{2}}}\right)^{D} \frac{1}{(1+\kappa)^{D}}\left(\frac{1}{r^{2}(\cos \theta+\sin \theta(\cos \varphi+\sin \varphi))^{2}}\right)^{\frac{D}{2}}
\end{aligned}
$$


The function $\frac{1}{(\cos \theta+\sin \theta(\cos \varphi+\sin \varphi))^{2}}$ varies between a minimum of $1 / 3$, when $\epsilon_{1}=\epsilon_{2}=\epsilon_{3}=\epsilon$ $(r=\sqrt{3}(1-\epsilon))$, and a maximum of 1 . Thus it is clear that for $\epsilon_{1}=\epsilon_{2}=\epsilon_{3}$ eq.(5.15) cannot be satisfied. On the other hand there are many ways to satisfy

$$
\frac{1}{\cos \theta+\sin \theta(\cos \varphi+\sin \varphi)}=\frac{1}{\cos \phi+\sin \phi}
$$

in which case (C.10) reduces to (C.7). The simplest way is to set $\varphi=0, \theta=\phi$ or $\theta=$ $\pi / 2, \varphi=\phi$. These correspond to ordered limits.

\section{Derivation of ghost product}

Here we sketch a derivation of the $*_{g}$ product of two states of the form (6.9) given in eq. 6.13). We need to calculate

$$
\left|\widetilde{\Xi}_{\tilde{\epsilon}}\right\rangle *_{g}\left|\widetilde{\Xi}_{\tilde{\eta}}\right\rangle={ }_{1}\left\langle\left.\widetilde{\Xi}_{\tilde{\epsilon}}\right|_{2}\left\langle\widetilde{\Xi}_{\tilde{\eta}} \mid \widetilde{V}_{3}\right\rangle\right.
$$

where the ghost part of the 3 -strings vertex $\left|\widetilde{V}_{3}\right\rangle$ is given in (6.2). Using the rules for bpz-conjugation we obtain

$$
\left\langle\widetilde{\Xi}_{\tilde{\epsilon}}\right|=\widetilde{N}_{\tilde{\epsilon}}\langle 0| c_{1}^{\dagger} e^{-c \widetilde{S}_{\tilde{\epsilon}} b} .
$$

Plugging this and ([6.2) in (D.1), and following the steps outlined in [13], one obtains

$$
\begin{aligned}
& \left|\widetilde{\Xi}_{\tilde{\epsilon}}\right\rangle *_{g}\left|\widetilde{\Xi}_{\tilde{\eta}}\right\rangle=\widetilde{\mathcal{N}}_{\tilde{\epsilon}} \widetilde{\mathcal{N}}_{\tilde{\eta}} \operatorname{det}\left(1-\widetilde{\mathcal{T}}_{\tilde{\epsilon} \tilde{\eta}} \widetilde{\mathcal{M}}\right) \\
& \quad \times\left\{1+c^{\dagger}\left[\widetilde{\mathbf{v}}_{0}+\left(\widetilde{V}_{+}, \widetilde{V}_{-}\right)\left(1-\widetilde{\mathcal{T}}_{\tilde{\epsilon} \tilde{\eta}} \widetilde{\mathcal{M}}\right)^{-1} \widetilde{\mathcal{T}}_{\tilde{\epsilon} \tilde{\eta}}\left(\begin{array}{c}
\widetilde{\mathbf{v}}_{+} \\
\widetilde{\mathbf{v}}_{-}
\end{array}\right)\right] b_{0}\right\} e^{c^{\dagger} C \widetilde{T}_{\tilde{\epsilon}} * \widetilde{T}_{\tilde{\eta}} b^{\dagger}} c_{0} c_{1}|0\rangle .
\end{aligned}
$$

Here the summations over mode indexes with positive values are understood. $\widetilde{\mathcal{T}}_{\tilde{\epsilon} \tilde{\eta}}$ and $\widetilde{\mathcal{M}}$ are defined as in (4.6) and (2.30), but with tildes. We also define

$$
\widetilde{T}_{\tilde{\epsilon}} * \widetilde{T}_{\tilde{\eta}}=\widetilde{X}+\left(\widetilde{X}_{+}, \widetilde{X}_{-}\right)\left(1-\widetilde{\mathcal{T}}_{\tilde{\epsilon} \tilde{\eta}} \widetilde{\mathcal{M}}\right)^{-1} \widetilde{\mathcal{T}}_{\tilde{\epsilon} \tilde{\eta}}\left(\begin{array}{c}
\widetilde{X}_{-} \\
\widetilde{X}_{+}
\end{array}\right) .
$$

Now one should observe that this formula is the same as (4.5), and as tilded matrices satisfy the same algebraic relations as the untilded ones, the result has the same form, i.e., we obtain

$$
\widetilde{T}_{\tilde{\epsilon}} * \widetilde{T}_{\tilde{\eta}}=\widetilde{T}_{\tilde{\epsilon} \star \tilde{\eta}} \cdot
$$

This constitutes, after using (B.20), the proof of eq. (6.11) for the reduced star product.

Now we must consider the part including the $b_{0}$ mode. After lengthier but straightforward manipulations, using the formulas from appendix A and sections 2, 3 and 4, we obtain that the expression inside square brackets in (D.3) can be written as

$$
\widetilde{\mathbf{v}}_{0}+\left(\widetilde{V}_{+}, \widetilde{V}_{-}\right)\left(1-\widetilde{\mathcal{T}}_{\tilde{\epsilon} \tilde{\eta}} \widetilde{\mathcal{M}}\right)^{-1} \widetilde{\mathcal{T}}_{\tilde{\epsilon} \tilde{\eta}}\left(\begin{array}{c}
\widetilde{\mathbf{v}}_{+} \\
\widetilde{\mathbf{v}}_{-}
\end{array}\right)=C\left(1-\widetilde{T}_{\tilde{\epsilon} \star \tilde{\eta}}\right) \mathbf{f},
$$


where $\mathbf{f}=\left\{f_{n}\right\}$ is the vector defined in appendix A. Using this in (D.3) we get

$$
\left|\widetilde{\Xi}_{\tilde{\epsilon}}\right\rangle *_{g}\left|\widetilde{\Xi}_{\tilde{\eta}}\right\rangle=\frac{\widetilde{\mathcal{N}}_{\tilde{\epsilon}} \widetilde{\mathcal{N}}_{\tilde{\eta}}}{\widetilde{\mathcal{N}}_{\tilde{\epsilon} \star \tilde{\eta}}}\left[\frac{1+(1-\tilde{\epsilon})(1-\tilde{\eta}) \widetilde{\kappa}}{\widetilde{\kappa}+1}\right]^{2} \operatorname{det}(1-\widetilde{T} \widetilde{\mathcal{M}})\left[c_{0}+c^{\dagger} C\left(1-\widetilde{T}_{\tilde{\epsilon} \star \tilde{\eta}}\right) \mathbf{f}\right]\left|\widetilde{\Xi}_{\tilde{\epsilon} \star \tilde{\eta}}\right\rangle .
$$

On the other hand, one easily shows that

$$
\mathcal{Q}\left|\widetilde{\Xi}_{\tilde{\epsilon}}\right\rangle=\left(c_{0}+\sum_{n=1}^{\infty} f_{n}\left(c_{n}+(-1)^{n} c_{n}^{\dagger}\right)\right)\left|\widetilde{\Xi}_{\tilde{\epsilon}}\right\rangle=\left[c_{0}+c^{\dagger} C\left(1-\widetilde{T}_{\tilde{\epsilon}}\right) \mathbf{f}\right]\left|\widetilde{\Xi}_{\tilde{\epsilon}}\right\rangle .
$$

Using this in (D.7) finally one gets (6.13).

\section{References}

[1] L. Rastelli, A. Sen and B. Zwiebach, "Vacuum string field theory," arXiv:hep-th/0106010.

[2] E.Witten, Noncommutative Geometry and String Field Theory, Nucl.Phys. B268 (1986) 253.

[3] A. Sen, "Rolling tachyon," JHEP 0204 (2002) 048 [arXiv:hep-th/0203211].

A. Sen, "Tachyon matter," JHEP 0207 (2002) 065 [arXiv:hep-th/0203265].

A. Sen, "Time evolution in open string theory," JHEP 0210 (2002) 003 [arXiv:hep-th/0207105].

A. Sen, "Time and tachyon," Int. J. Mod. Phys. A 18 (2003) 4869 [arXiv:hep-th/0209122].

A. Sen, "Open and closed strings from unstable D-branes," arXiv:hep-th/0305011.

A. Sen, "Open-closed duality: Lessons from matrix model," [arXiv:hep-th/0308068].

D. Gaiotto, N. Itzhaki and L. Rastelli, "Closed strings as imaginary D-branes," [arXiv:hep-th/0304192].

I. R. Klebanov, J. Maldacena and N. Seiberg, "D-brane decay in two-dimensional string theory," JHEP 0307 (2003) 045 [arXiv:hep-th/0305159].

N. Lambert, H. Liu and J. Maldacena, "Closed strings from decaying D-branes," arXiv:hep-th/0303139.

[4] J. Kluson, "Time dependent solution in open bosonic string field theory,"

arXiv:hep-th/0208028. M. Fujita and H. Hata, "Time dependent solution in cubic string field theory," JHEP 0305 (2003) 043 [arXiv:hep-th/0304163].

[5] V.A.Kostelecky and R.Potting, Analytical construction of a nonperturbative vacuum for the open bosonic string, Phys. Rev. D 63 (2001) 046007 [hep-th/0008252].

[6] L.Rastelli, A.Sen and B.Zwiebach, String field theory around the tachyon vacuum, Adv. Theor. Math. Phys. 5 (2002) 353 [hep-th/0012251].

[7] L.Rastelli, A.Sen and B.Zwiebach, Classical solutions in string field theory around the tachyon vacuum, Adv. Theor. Math. Phys. 5 (2002) 393 [hep-th/0102112].

[8] L.Rastelli, A.Sen and B.Zwiebach, Half-strings, Projectors, and Multiple D-branes in Vacuum String Field Theory, JHEP 0111 (2001) 035 [hep-th/0105058].

[9] T.Okuda, The Equality of Solutions in Vacuum String Field Theory, Nucl. Phys. B641 (2002) 393 [hep-th/0201149].

[10] D. Gaiotto, L. Rastelli, A. Sen and B. Zwiebach, Star Algebra Projectors, JHEP 0204 (2002) 060 [hep-th/0202151]. 
[11] G.Moore and W.Taylor The singular geometry of the sliver, JHEP 0201 (2002) 004 [hep-th/0111069].

[12] N. Moeller, "Some exact results on the matter star-product in the half-string formalism," JHEP 0201 (2002) 019 [arXiv:hep-th/0110204].

[13] H.Hata and T.Kawano, Open string states around a classical solution in vacuum string field theory, JHEP 0111 (2001) 038 [hep-th/0108150].

[14] H.Hata and S.Moriyama, Observables as Twist Anomaly in Vacuum String Field Theory, JHEP 0201 (2002) 042 [hep-th/0111034].

[15] H. Hata, S. Moriyama and S. Teraguchi, "Exact results on twist anomaly," JHEP 0202 (2002) 036 [arXiv:hep-th/0201177].

[16] H.Hata and S.Moriyama, Reexamining Classical Solution and Tachyon Mode in Vacuum String Field Theory, Nucl. Phys. B 651 (2003) 3 [hep-th/0206208].

[17] L.Rastelli, A.Sen and B.Zwiebach, A note on a Proposal for the Tachyon State in Vacuum String Field Theory, JHEP 0202 (2002) 034 [hep-th/0111153].

[18] H.Hata and H.Kogetsu Higher Level Open String States from Vacuum String Field Theory, JHEP 0209 (2002) 027, [hep-th/0208067].

[19] J.R.David, Excitations on wedge states and on the sliver, JHEP 0107 (2001) 024 [hep-th/0105184].

[20] Y.Okawa, Open string states and D-brane tension form vacuum string field theory, JHEP 0207 (2002) 003 [hep-th/0204012].

[21] D.Gaiotto, L.Rastelli, A.Sen and B.Zwiebach, Ghost Structure and Closed Strings in Vacuum String Field Theory, [hep-th/0111129].

[22] L.Rastelli, A.Sen and B.Zwiebach, Star Algebra Spectroscopy, JHEP 0203 (2002) 029 [hep-th/0111281].

[23] M.Schnabl, Wedge states in string field theory, JHEP 0301 (2003) 004, [hep-th/0201095]. Anomalous reparametrizations and butterfly states in string field theory, Nucl. Phys. B 649 (2003) 101, [hep-th/0202139].

[24] D.J.Gross and A.Jevicki, Operator Formulation of Interacting String Field Theory, Nucl.Phys. B283 (1987) 1.

[25] D.J.Gross and A.Jevicki, Operator Formulation of Interacting String Field Theory, 2, Nucl.Phys. B287 (1987) 225.

[26] N. Ohta, "Covariant Interacting String Field Theory In The Fock Space Representation," Phys. Rev. D 34 (1986) 3785 [Erratum-ibid. D 35 (1987) 2627].

[27] L. Bonora, C. Maccaferri, D. Mamone and M. Salizzoni, "Topics in string field theory," arXiv:hep-th/0304270.

[28] A.Leclair, M.E.Peskin, C.R.Preitschopf, String Field Theory on the Conformal Plane. (I) Kinematical Principles, Nucl.Phys. B317 (1989) 411.

[29] A.Leclair, M.E.Peskin, C.R.Preitschopf, String Field Theory on the Conformal Plane. (II) Generalized Gluing, Nucl.Phys. B317 (1989) 464. 
[30] S. Samuel, The Ghost Vertex In E. Witten's String Field Theory, Phys. Lett. B 181 (1986) 255.

[31] K.Okuyama, Ratio of Tensions from Vacuum String Field Theory, JHEP 0203 (2002) 050 [hep-th/0201136].

[32] K.Okuyama, Siegel Gauge in Vacuum String Field Theory, JHEP 0201 (2002) 043 [hep-th/0111087]. I.Kishimoto, Some properties of string field algebra, JHEP 0112 (2001) 007 [hep-th/0110124].

[33] C. Maccaferri and D. Mamone, "Star democracy in open string field theory," JHEP 0309 (2003) 049 [arXiv:hep-th/0306252].

[34] K.Okuyama, Ghost Kinetic Operator of Vacuum String Field Theory, JHEP 0201 (2002) 027 [hep-th/0201015].

[35] D.M. Belov, A. Konechny, On spectral density of Neumann matrices, Phys.Lett. B558 (2003) 111-118 [hep-th/0210169]. D.M.Belov, Diagonal Representation of Open String Star and Moyal Product, [hep-th/0204164]. D.M.Belov, Witten's Ghost Vertex Made Simple (bc and bosonized ghosts), [hep-th/0308147] E. Fuchs, M. Kroyter and A. Marcus, "Virasoro operators in the continuous basis of string field theory," JHEP 0211 (2002) 046 [arXiv:hep-th/0210155].

[36] L. Bonora, D. Mamone and M. Salizzoni, B field and squeezed states in Vacuum String Field Theory, Nucl. Phys. B630 (2002) 163 [hep-th/0201060].

[37] L. Bonora, D. Mamone and M. Salizzoni, Vacuum String Field Theory with B field, JHEP 0204 (2002) 020 [hep-th/0203188].

[38] L. Bonora, D. Mamone and M. Salizzoni, Vacuum String Field Theory ancestors of the GMS solitons, JHEP 0301 (2003) 013 [hep-th/0207044].

[39] B. Feng, Y. H. He and N. Moeller, "The spectrum of the Neumann matrix with zero modes," JHEP 0204 (2002) 038 [arXiv:hep-th/0202176].

[40] Y. Okawa, "Some exact computations on the twisted butterfly state in string field theory," [arXiv:hep-th/0310264.]

[41] L. Bonora, C. Maccaferri, P. Prester, in preparation 